\title{
Article \\ Dynamic Disturbance and Error Analysis of Flexible Support System for Large Optical Mirror Processing
}

\author{
Zujin Jin ${ }^{1}{ }^{1}$, Gang Cheng ${ }^{1, *}$, Shichang $X u^{1}$ and Wei Gu ${ }^{2}$ \\ 1 School of Mechatronic Engineering, China University of Mining and Technology, Xuzhou 221116, China; \\ jinzjcam@cumt.edu.cn (Z.J.); TB19050022B2@cumt.edu.cn (S.X.) \\ 2 Shangdong Zhongheng Optoelectronic Technology Co., Ltd., Zaozhuang 277000, China; \\ Hebutguwei@163.com \\ * Correspondence: chg@cumt.edu.cn; Tel.: +86-132-2523-2379
}

check for updates

Citation: Jin, Z.; Cheng, G.; Xu, S.; $\mathrm{Gu}$, W. Dynamic Disturbance and Error Analysis of Flexible Support System for Large Optical Mirror Processing. Appl. Sci. 2021, 11, 2715. https://doi.org/10.3390/ app11062715

Academic Editor: Paolo Renna

Received: 28 February 2021

Accepted: 15 March 2021

Published: 18 March 2021

Publisher's Note: MDPI stays neutral with regard to jurisdictional claims in published maps and institutional affiliations.

Copyright: (c) 2021 by the authors Licensee MDPI, Basel, Switzerland. This article is an open access article distributed under the terms and conditions of the Creative Commons Attribution (CC BY) license (https:// creativecommons.org/licenses/by/ $4.0 /)$.
Featured Application: This study adopts the method of mathematical model-simulation-experiment to study the non-linear influence relationship between the ambient temperature and the air content in the working medium. The parameters of the model are modified by simulation analysis and combined with experimental parameters to verify that the established mathematical model can be consistent with the actual working conditions. The established mathematical model provides a theoretical basis for the subsequent modal analysis of the flexible support system (FSS) and provides an experimental basis for the design of the controller and the study of error compensation control strategies. At the same time, this method can be extended to studies on the error mechanisms of other nonlinear, time-varying, uncertain, and externally disturbed systems.

Abstract: To improve the accuracy of a flexible support system (FSS) used for optical mirror processing, the influence of air content in the working medium and ambient temperature change on the FSS is analyzed and studied. First, the disturbance model of the FSS and single support cylinder affected by different air contents in the working medium and ambient temperature is established, and the mapping relationship between the influencing factors and the affected factors is analyzed. Then, the effects of ambient temperature change on volume, support height, and support pressure for different air contents are simulated and analyzed separately. The results of the simulation obtained show that when the working medium is mixed with different volume fractions of air and the ambient temperature changes, upper and lower chamber volumes, support rigidity, and support height of the support cylinder are also changed. Finally, an experimental study of pressure changes in the upper and lower chambers, support height, and support rigidity changes at different ambient temperatures and air contents are carried out. By measuring the support height, support pressure, and support rigidity error, the effectiveness of the established mathematical disturbance model of FSS is further verified. It not only provides a theoretical basis for improving the support accuracy of the FSS but also provides a foundation for the application of the FSS in the processing stage of large optical mirrors.

Keywords: optical mirror processing; flexible support system; dynamic disturbance; elastic modulus; error analysis

\section{Introduction}

With the information revolution, modern optical mirrors are being developed for large apertures and high precision [1-3]. These large apertures and precision requirements have increased the difficulty of processing, and the requirements for the processing equipment have also increased [4]. During optical mirror surface processing, the processing robot drives a polishing disc to reach the grinding residence point of the optical mirror surface [5-7]. At the same time, the bottom support system should realize the real-time adjustment of the height and posture of the supported mirror and its supporting stiffness. To improve the 
accuracy of the optical mirror processing system, in addition to improving the motion accuracy and repeated positioning accuracy of the processing robot, the performance parameters of the support system are the main factors that determine the precision of the optical mirror surface. Traditional optical mirror support systems mostly offer base support, mechanical whiffle-tree support, and endless belt support [8]. They can meet the rigid requirements of an optical mirror support system, but due to the non-adjustability of the support stiffness, it is difficult or even impossible to unload the impact load produced by the tool in the process of machining. It may damage the surface of the optical mirror to be processed, especially for large, lightweight, and thin mirrors $[9,10]$. It may crush the mirror surface, resulting in a large amount of manpower and material resources.

In the research of an optical mirror flexible support system (FSS), some scholars used pneumatic support, hydraulic support, or other forms of FSS for the postural adjustment and error correction of optical mirrors and achieved good results. To improve the fabrication efficiency and testing accuracy of the meter-scale, Hu et al. designed and manufactured dozens of hydraulic support units and tested them through a $4 \mathrm{~m} \mathrm{SiC} \mathrm{mirror,} \mathrm{which} \mathrm{proved}$ the high accuracy of the designed support system [11]. To release the thermal stress of the optical primary mirror, Huo et al. proposed a novel kinematic flexure mount composed of three identical chains and analyzed the stiffness characteristics of its structure [12]. Yu et al. designed a new type of three-leaf flexible structure based on the principle of spring, and optimized the size parameters of the flexible structure through the finite element analysis method [13]. In order to achieve accurate surface shape and optical axis stability of a large aperture lens in a ground-based telescope system that is subject to various load cases, Zhang et al. proposed a novel lens support with a multi-point flexible support structure and optimized the support structure based on low-order modes and system accuracy [14]. The above scholars have conducted a lot of research on the support system of optical mirrors. They used support systems for the installation, positioning, and detection of large-diameter or thin mirrors by improving traditional mechanisms, designing new support systems, and optimizing the mechanism parameters of the support systems. However, only a few scholars applied the FSS to the optical mirror processing stage and conducted in-depth research on the FSS. Therefore, it is necessary to further study the application of the FSS in the processing stage of large-aperture optical mirrors. The FSS is uncertain, non-linear, and susceptible to external disturbances, so it is relatively difficult to control and easily produces errors $[15,16]$. To ensure the stability of the support stiffness, support height, and output force accuracy of the FSS, it is necessary to analyze the degree of influence of the FSS on the external environment.

Several scholars have done a lot of research on the influence of external environmental disturbances on nonlinear hydraulic systems. Gholizadeh et al. analyzed the number of air bubbles entrained in hydraulic oil, the size and distribution of these bubbles, and the influence of the compressibility of the mixture on the effective bulk modulus and proposed a new theoretical model [17]. To study the low-pressure effective volume of hydraulic oil, Kim et al. calculated the effective bulk elastic modulus by three different methods: mass change, volume change, and sound speed method, and determined the relationship between the release pressure of dissolved air in the oil and the variable gas constant with the rate of volume change [18]. Through the combination of experimental and mathematical model methods, Burecek et al. determined the undissolved air content in hydraulic oil and calculated the bulk modulus of hydraulic oil considering the hydraulic oil system in a pipeline [19]. Zhou et al. addresses the problem of bubble evolution arising from gas cavitation in hydraulic oils, including the interphase mass transfer represented by air release and absorption phenomena and different thermodynamic considerations, and three new models in a progressive relationship were proposed on the basis of the Rayleigh-Plesset equation which describes bubble dynamics [20]. Mobayen et al. designed a robust controller for uncertain systems with time-varying uncertainties, nonlinearities, and external disturbances to reduce the influence of external disturbances on the system and improve system stability [21,22]. Based on an improvement of Henry's law and the 
polytropic process equation for gases, Yuan et al. proposed a modified model for the dynamic bulk modulus of gas-containing hydraulic oil. Through the analysis of parameter influence, the influence of initial gas content and pressure on the dynamic bulk modulus of gas-containing hydraulic oil during compression and expansion was discussed [23]. Based on a modified effective bulk modulus model of hydraulic oil built upon the IFAS model (developed at the Institute fur Fluidtechnische Antriebe and Steuerungen, RheinischWestfälische Technische Hochschule Aachen university), Righettini et al. developed an empirical non-linear model for a servo-hydraulic uni-axial shaking table [24]. The above scholars have analyzed the elastic modulus and the change in stiffness of hydraulic oil with the air content and temperature. The theoretical models for different working conditions were respectively established, and the mode accuracy was verified. However, there is little research on the influence of the ambient temperature and working medium on the support pressure, support stiffness, and support height of the FSS used for large-scale optical mirror processing support.

To achieve adjustable support stiffness, a flexible support system for an optical mirror is proposed, which uses hydraulic oil as the working medium. It could unload the impact force exerted by robots on the mirror to be machined effectively. The support pressure, support height, and support stiffness of the FSS are easily affected by the ambient temperature and working medium, and the influence relationship is time-varying and nonlinear. This leads to poor stability of the FSS, which in turn affects the accuracy of the optical mirror. Therefore, it is necessary to study and analyze the changes in performance parameters of the FSS at different temperatures and air contents of the working medium. According to the structural parameters of the FSS, a nonlinear mathematical model of the FSS is established. This model can reflect the influence of ambient temperature and air content in the working medium on the FSS. Through the simulation of the mathematical model, the mapping relationship between the influencing factors and affected factors is analyzed. Finally, combined with relevant experimental analysis, the validity of the established model is verified. It provides a theoretical basis for improving the accuracy of the FSS and provides a research foundation for the application of the FSS in the processing of large optical mirrors. The established mathematical model provides a theoretical basis for the subsequent modal analysis of the FSS and provides an experimental basis for the design of the controller and the study of error compensation control strategies. The mathematical model-simulationexperiment method used in this study examines the relationship between the performance parameters of the support system and the air content in the working medium, thereby providing a theoretical framework for the analysis of time-varying uncertainty, nonlinearity, and external interference research.

\section{Establishment of Disturbance Model}

\subsection{Establishment of Disturbance Model of FSS}

The 3D model of FSS is shown in Figure 1a. It consists of 36 support cylinders, which are evenly distributed on the support floor with a diameter of $1250 \mathrm{~mm}$. As shown in Figure 1b, the FSS is divided into three sectors on average, and each sector contains 12 support cylinders. The upper and lower chambers of support cylinders in each sector are connected through hydraulic lines, which ensures that the performance parameters of the upper and lower chambers in the same sector are the same. The support rigidity of the support cylinder can be adjusted by changing the pressure value of the upper and lower chambers, and the support height of support cylinder can be adjusted by changing the pressure difference of the upper and lower chambers. Therefore, the attitude of the supported optical mirror can be finely adjusted by changing the support height and support rigidity of three sectors of FSS. During the processing of the optical mirror, the change of ambient temperature and the air contained in the working medium will affect the support pressure, support rigidity, and support height of FSS. To study the influence level of each influencing factor and facilitate the error analysis of each influencing link, the disturbance model of FSS for the ambient temperature and working medium was established. 


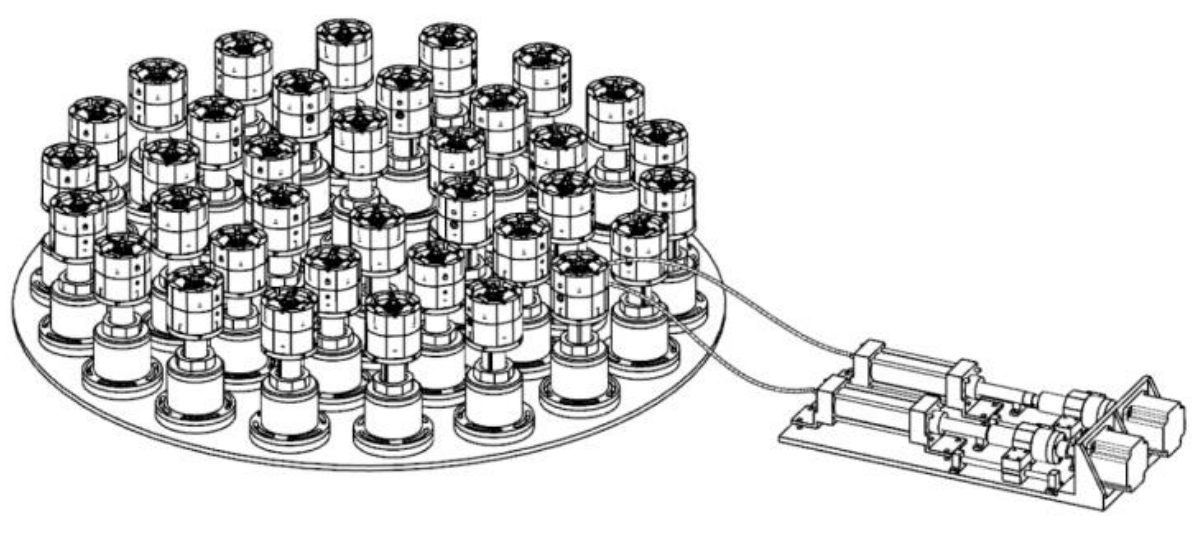

(a)

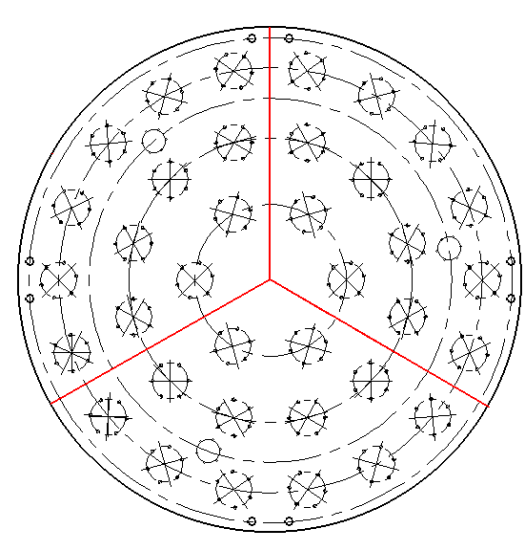

(b)

Figure 1. Model of the flexible support system (FSS). (a) 3D model of the FSS. (b) Arrangement of support cylinder.

Because of the independence of the three working sectors of the FSS, the working pressure and air content of the working medium in the sectors are not identical and do not interfere with each other. The disturbance model of the flexible support cylinder can also be divided into three parts:

$$
\boldsymbol{D}=\left[\begin{array}{l}
\boldsymbol{D}_{1} \\
\boldsymbol{D}_{2} \\
\boldsymbol{D}_{3}
\end{array}\right]=\left[\begin{array}{c}
\sum_{i=1}^{12}\left(a_{i} f\left(P, F, \Delta \boldsymbol{T}_{i}\right)+b_{i} f\left(P, F, \Delta \boldsymbol{V}_{i}\right)+\boldsymbol{M}_{i}\right) \\
\sum_{j=1}^{12}\left(a_{j} f\left(P, F, \Delta \boldsymbol{T}_{j}\right)+b_{j} f\left(P, F, \Delta \boldsymbol{V}_{j}\right)+\boldsymbol{M}_{j}\right) \\
\sum_{k=1}^{12}\left(a_{k} f\left(P, F, \Delta \boldsymbol{T}_{k}\right)+b_{k} f\left(P, F, \Delta \boldsymbol{V}_{k}\right)+\boldsymbol{M}_{k}\right)
\end{array}\right]
$$

where $D_{1}$ is the disturbance of sector $1, D_{2}$ is the disturbance of sector $2, D_{3}$ is the disturbance of sector 3, $T$ is the disturbance of ambient temperature, $V$ is the disturbance of working medium, $a, b$ are disturbance coefficients, $\boldsymbol{M}$ is the disturbance caused by the manufacturing error of the parts, $P$ is the working pressure, and $F$ is the force at the support point.

\subsection{Disturbance Model of Single Support Cylinder}

\subsubsection{Support Cylinder Structure}

The support cylinder is composed of two chambers: an upper chamber and a lower chamber. The structures of the support cylinder are shown in Figure 2.

The movement of the support cylinder can be expressed as

$$
\Delta L=\frac{\Delta V_{u}-\Delta V_{d}}{2 \cdot S}
$$

where $\Delta L$ is the displacement of support cylinder, $\Delta V_{u}$ is the volume change of the upper chamber, $\Delta V_{d}$ is the volume change of the lower chamber, $S$ is the cross-sectional area of support piston. 


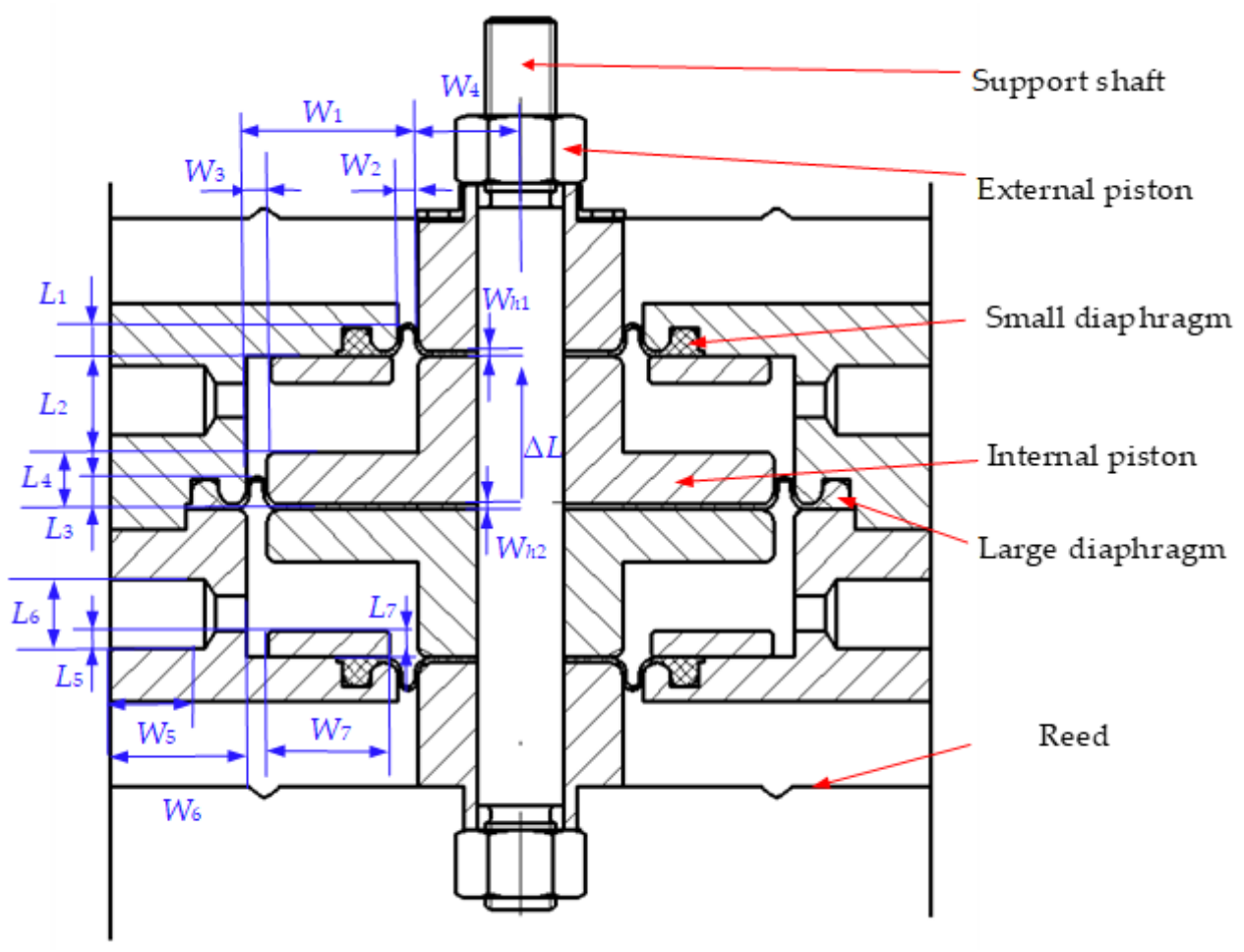

Figure 2. Structure of support cylinder.

When the support shaft of support cylinder moves $\Delta L$, the volume of the upper and lower chambers can be expressed as:

$$
\begin{aligned}
& V_{u}=f\left(\boldsymbol{W}, \boldsymbol{L}, \Delta L, \Delta L_{1}, \Delta L_{3}, \Delta L_{4}, \Delta L_{6}, R_{p}\right) \\
& V_{d}=f\left(\boldsymbol{W}, \boldsymbol{L}, \Delta L, \Delta L_{2}, \Delta L_{3}, \Delta L_{5}, \Delta L_{6}, R_{p}\right)
\end{aligned}
$$

where $\boldsymbol{W}=\left[\begin{array}{ccc}W_{1} & & \\ & \ddots & \\ & & W_{m}\end{array}\right]$ and $\boldsymbol{L}=\left[\begin{array}{ccc}L_{1} & & \\ & \ddots & \\ & & L_{n}\end{array}\right]$ are the structural parameters of support cylinder, $m$ and $n$, respectively, indicate the number of radial and axial structural parameters of support cylinder, $\Delta L_{1}$ is the extension quantity of the small rolling diaphragm in upper chamber, $\Delta L_{2}$ is the extension quantity of the small rolling diaphragm in lower chamber, $\Delta L_{3}$ is the extension quantity of the large rolling diaphragm, $\Delta L_{4}$ is the compression quantity of the small rolling diaphragm in upper chamber, $\Delta L_{5}$ is the compression quantity of the small rolling diaphragm in lower chamber, $\Delta L_{6}$ is the compression quantity of the large rolling diaphragm, $R_{p}$ is the chamfer radius of the chamber.

During the work of the support cylinder, the hydraulic oil in the upper chamber exerts a force on the small rolling diaphragm in the upper chamber and the large rolling diaphragm, and the hydraulic oil in the lower chamber will exert a force on the small rolling diaphragm in the lower chamber and the large rolling diaphragm. Because the pressures in the upper and lower chambers of the support cylinder, as well as the deformation of the small rolling diaphragm in the upper and lower chambers, are different, the deformations of the rolling diaphragms in the upper and lower chambers need to be calculated separately.

(1) Deformations of the small rolling diaphragms in the upper and lower chambers are:

$$
\begin{aligned}
& \Delta L_{1}=\frac{W_{2} L_{1}}{2 E W_{h 1}} P_{u} \\
& \Delta L_{2}=\frac{W_{2} L_{1}}{2 E W_{h 1}} P_{d}
\end{aligned}
$$


where $P_{u}$ is the pressure of upper chamber, $P_{d}$ is the pressure of lower chamber, $E$ is the modulus of elasticity, $W_{h 1}$ is the thickness of small rolling diaphragm, $W_{2}$ is the gap between outer piston and cylinder wall, $L_{1}$ is the mounting height of small rolling diaphragm.

(2) Deformation of the large rolling diaphragm can be expressed as:

$$
\Delta L_{3}=\frac{W_{3} L_{3}}{2 E W_{h 2}}\left(P_{u}-P_{d}\right)
$$

where $W_{h 2}$ is the thickness of large rolling diaphragm, $W_{3}$ is the gap between internal piston and cylinder wall, $L_{3}$ is the installation height of large rolling diaphragm.

(3) Compression of the rolling diaphragm

The compression process of the rolling diaphragm, owing to the relatively small amount of deformation, can be simplified to a simple compression deformation.

$$
P_{1} \cdot S_{p}=E \cdot S_{p} \cdot \varepsilon
$$

where $\sigma$ is the stress on rolling diaphragm, $P_{1}$ is the pressure on rolling diaphragm, $S_{p}$ is the area under compression, $\varepsilon$ is the unit deformation. Through Equation (8), the deformation per unit thickness of the small rolling diaphragms of upper and lower chambers and the large diaphragm can be calculated.

$$
\begin{aligned}
& \Delta L_{4}=\varepsilon_{1} * W_{h 1} \\
& \Delta L_{5}=\varepsilon_{2} * W_{h 1} \\
& \Delta L_{6}=\varepsilon_{3} * W_{h 2}
\end{aligned}
$$

where $\varepsilon_{1}$ is the amount of deformation per unit thickness of the small rolling diaphragm in the upper chamber, $\varepsilon_{2}$ is the amount of deformation per unit thickness of the small rolling diaphragm in the lower chamber, $\varepsilon_{3}$ is the amount of deformation per unit thickness of the large rolling diaphragm.

\subsubsection{Working Medium}

The working medium used in the FSS is hydraulic oil, which has fixed physical properties at an ambient temperature and pressure, mainly the compressibility and expansion. The state equation of hydraulic oil can be expressed as

$$
f(\rho, P, T)=0
$$

where $\rho$ is the density of hydraulic oil, $P$ is the pressure of hydraulic oil, $T$ is the temperature of hydraulic oil.

The state equation can be Taylor expanded at $\rho_{0}$.

$$
\begin{gathered}
\rho=\rho_{0}\left(P_{0}, T_{0}\right)+\left.\frac{\partial \rho(P, T)}{\partial P}\right|_{T_{0}}\left(P-P_{0}\right)+\left.\frac{\partial \rho(P, T)}{\partial T}\right|_{P_{0}}\left(T-T_{0}\right)+\left.\frac{1}{2 !} \frac{\partial^{2} \rho(P, T)}{\partial^{2} P}\right|_{T_{0}}\left(P-P_{0}\right)^{2}+ \\
\left.\frac{1}{2 !} \frac{\partial^{2} \rho(P, T)}{\partial P \partial T}\right|_{T_{0}, P_{0}}\left(P-P_{0}\right)\left(T-T_{0}\right)+\left.\frac{1}{2 !} \frac{\partial^{2} \rho(P, T)}{\partial T \partial P}\right|_{P_{0}, T_{0}}\left(P-P_{0}\right)\left(T-T_{0}\right)+\left.\frac{1}{2 !} \frac{\partial^{2} \rho(P, T)}{\partial^{2} T}\right|_{P_{0}}\left(T-T_{0}\right)^{2}+\cdots+o^{n}
\end{gathered}
$$

where $o^{n}$ is the Lagrange remainder.

$$
o^{n}=\frac{1}{(n+1) !}\left(\frac{\partial \rho(P, T)}{\partial P}+\frac{\partial \rho(P, T)}{\partial T}\right)^{n+1} \rho\left(P_{0}+\theta P, T_{0}+\theta T\right)
$$

where $0<\theta<1$.

Written in incremental form:

$$
\begin{gathered}
\rho-\rho_{0}\left(P_{0}, T_{0}\right)=d \rho=\left.\frac{\partial \rho(P, T)}{\partial P}\right|_{T_{0}} d P+\left.\frac{\partial \rho(P, T)}{\partial P}\right|_{P_{0}} d T+\left.\frac{1}{2 !} \frac{\partial^{2} \rho(P, T)}{\partial^{2} P}\right|_{T_{0}} d^{2} P \\
+\left.\frac{1}{2 !} \frac{\partial^{2} \rho(P, T)}{\partial P \partial T}\right|_{T_{0}, P_{0}} d P d T+\left.\frac{1}{2 !} \frac{\partial^{2} \rho(P, T)}{\partial T \partial P}\right|_{P_{0}, T_{0}} d T d P+\left.\frac{1}{2 !} \frac{\partial^{2} \rho(P, T)}{\partial^{2} T}\right|_{P_{0}} d^{2} T+\cdots+o^{n}
\end{gathered}
$$


Owing to the compression of density relative to pressure and the difficulty in measuring temperature, a relative quantity is used for analysis and calculation:

$$
\frac{d \rho}{\rho_{0}}=k d P+\alpha_{o i l} d T+\frac{1}{2 !} k^{2} d^{2} P+\frac{1}{2 !} k \alpha_{o i l} d P d T+\frac{1}{2 !} k \alpha_{o i l} d T d P+\frac{1}{2 !} \alpha_{o i l}^{2} d^{2} T+\cdots+\frac{1}{(n+1) !}\left(\alpha_{o i l}+k\right)^{n+1}
$$

where $k$ is the volume compression coefficient of hydraulic oil, and $\alpha_{\text {oil }}$ is the expansion of hydraulic oil.

(1) Expansion of hydraulic oil

Assuming that the volume of hydraulic oil is $V_{0}$ at $0{ }^{\circ} \mathrm{C}$ and the volume is $V_{T}$ at $T{ }^{\circ} \mathrm{C}$, then the volume expansion coefficient is:

$$
V_{T}=V_{0}(1+\alpha T)
$$

Because the volume expansion coefficient of hydraulic oil is very small, for the convenience of calculation when the temperature is not high, the following equation can be directly used for calculation: not taking $V_{0}$ into consideration at $0{ }^{\circ} \mathrm{C}, \alpha$ is the volume expansion coefficient.

$$
V_{2}=V_{1}\left[1+\alpha\left(T_{2}-T_{1}\right)\right]
$$

When the hydraulic oil contains other substances:

$$
V_{t}=V_{1}\left[1+\alpha_{1}\left(T_{2}-T_{1}\right)\right]+V_{2}\left[1+\alpha_{2}\left(T_{2}-T_{1}\right)\right]+\cdots+V_{n}\left[1+\alpha_{n}\left(T_{2}-T_{1}\right)\right]
$$

where $V_{1}, V_{2}, \ldots, V_{n}$ is the volume fraction of the components of mixed liquid at a temperature of $T_{1}, \alpha_{1}, \alpha_{2}, \ldots, \alpha_{n} \alpha_{n}$ is the average value of volume expansion coefficients of the mixed liquid components when the temperature rises from $T_{1}$ to $T_{2}$.

(2) Compressibility of hydraulic oil

When hydraulic oil is mixed with air, the influence of the dissolved air on the elastic modulus can be ignored, and the air in the form of bubbles will affect the effective volume elastic modulus of hydraulic oil. Because the support cylinder is in a low-pressure working state, in order to facilitate the calculation, the following assumptions are made for the derivation of the theoretical model: (i) The variation of the hydraulic line with changes in temperature and pressure change may be ignored. (ii) The quality of air contained in hydraulic oil does not change with time. (iii) Because the support cylinder operates at a low pressure, the separation and dissolution of air in hydraulic oil during the pressure change can be ignored. (iv) The air contained in hydraulic oil is regarded as an ideal gas, which satisfies the ideal gas state equation.

When hydraulic oil is mixed with air, the density of the mixed medium is:

$$
\rho_{a}=\frac{m_{\text {oil }}+m_{\text {air }}}{V_{\text {oil }}+V_{\text {air }}}
$$

where $m_{\text {oil }}$ is the mass of hydraulic oil, $m_{\text {air }}$ is the mass of mixed air, $V_{\text {air }}$ is the volume of air content in working medium, $V_{\text {oil }}$ is the volume of hydraulic oil.

$$
V_{\text {oil }}=\frac{m_{\text {oil }}}{\rho_{r 1}\left[1+(1 / K)\left(P-P_{r 1}\right)+\alpha_{o i l}\left(T-T_{r 1}\right)\right]}
$$

where $K$ is the elastic modulus of pure hydraulic oil, $\rho_{r 1}$ is the calibrated density of pure hydraulic oil, $P_{r 1}$ is the calibrated pressure of pure hydraulic oil, $T_{r 1}$ is the calibrated temperature of pure hydraulic oil.

According to Equations (17) and (18), the comprehensive density of mixed hydraulic oil can be obtained as:

$$
\rho_{r}=\frac{m_{\text {oil }}+m_{\text {air }}}{m_{\text {oil }}+V_{\text {air }} \cdot \rho_{r 1}\left[1+\left(P-P_{r 1}\right) / K+\alpha_{o i l}\left(T-T_{r 1}\right)\right]}
$$




$$
k=\frac{1}{\rho_{r}} \frac{d\left(\rho_{r}\right)}{d P}
$$

According to the equation of the volume elastic coefficient, the volume compression coefficient of hydraulic oil can be expressed as:

$$
k=-\frac{1}{\Delta p} \cdot \frac{\Delta V}{V}
$$

Then, when the temperature and air content change, the change in pressure is:

$$
\Delta p=-\frac{1}{k} \cdot \frac{\Delta V}{V}
$$

\subsubsection{Support Stiffness}

When the support pressure of the upper and lower chambers and the volume parameters of the upper and lower chambers change, the support stiffness of the FSS changes.

$$
C_{K}=C_{1}+C_{2}+2 C_{p}+C_{B l}+2 C_{B s}
$$

where $C_{k}$ is the total stiffness of the support cylinder, $C_{1}$ is the stiffness of the upper chamber, $C_{2}$ is the stiffness of the lower chamber, $C_{p}$ is the stiffness of the beryllium bronze reed, $C_{B l}$ is the stiffness of the small diaphragm, $C_{B S}$ is the stiffness of the large diaphragm.

$$
\begin{aligned}
& C_{1}=\frac{\Delta P_{u} S}{\Delta L} \\
& C_{2}=\frac{\Delta P_{S} S}{\Delta L}
\end{aligned}
$$

where $\Delta P_{u}$ is the pressure change in the upper chamber, and $\Delta P_{s}$ is the pressure change in the lower chamber.

Based on the above, the relationship between temperature change and FSS performance under different air contents is shown in Figure 3.

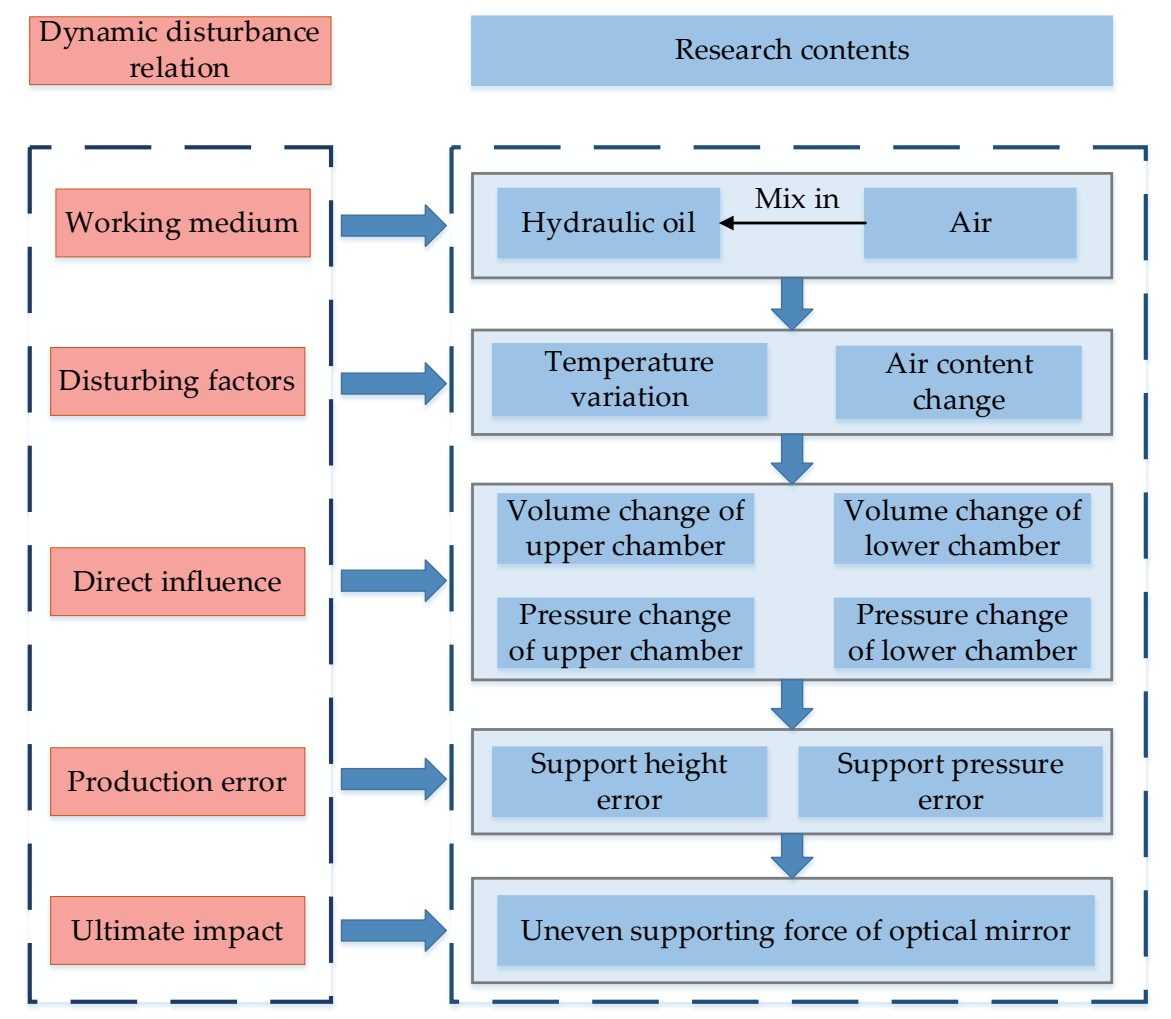

Figure 3. Perturbation affecting relationship. 
As shown in Figure 3, the hydraulic oil in the FSS contains air. During the working process, the change in ambient temperature will cause a change in the volume and pressure of the upper and lower chambers of the support cylinder. The air content in the working medium affects the change in volume and pressure. When the support shaft assumes different working positions, owing to the different volume capacities of the upper and lower chambers, the volume and pressure changes of the upper and lower chambers are also different. Changes in the volume and pressure of the upper and lower chambers of the support cylinder will cause changes in the support height, support pressure, and support stiffness, which will lead to the deformation of the supported optical mirror and affect the surface forming accuracy of the optical mirror surface.

\section{Simulation Analysis of FSS}

Under different FSS working pressures, the amount of air contained in the working medium and the change in ambient temperature during the work will have different degrees of influence on the support pressure and support height of the FSS. To study the corresponding relationship between the influencing factors and affected parameters, the effects of ambient temperature change on the volume, support height, and support pressure at different air contents are simulated.

In the simulation calculation process, the working medium characteristic parameters and disturbance coefficient are shown in Table 1.

Table 1. The working medium characteristic parameters and disturbance coefficient.

\begin{tabular}{cccc}
\hline Name & Value & Name & Value \\
\hline $\begin{array}{c}\text { Calibration temperature of } \\
\text { hydraulic oil }\end{array}$ & $T_{r 1}=20^{\circ} \mathrm{C}$ & The expansion of hydraulic oil & $\alpha_{\text {oil }}=1.66 \times 10^{-4}$ \\
$\begin{array}{c}\text { Elastic modulus of hydraulic } \\
\text { oil volume }\end{array}$ & $K=1.66 \times 10^{3} \mathrm{MPa}$ & The expansion of air & $\alpha_{\text {air }}=3.767 \times 10^{-3}$ \\
Density of hydraulic oil & $\rho_{r 1}=900 \mathrm{~kg} / \mathrm{m}^{3}$ & Density of air at $0{ }^{\circ} \mathrm{C}$ & $\rho_{r 2}=1.29 \mathrm{~kg} / \mathrm{m}^{3}$ \\
Standard atmosphere & $P_{r 1}=0.101 \mathrm{MPa}$ & Disturbance coefficient & $\mathrm{a}, \mathrm{b}=1$ \\
$\begin{array}{c}\text { The volume compression } \\
\text { coefficient of hydraulic oil }\end{array}$ & $k=1.17 \times 10^{-3}$ & Air content & $\Delta V=0-10 \%$ \\
\hline
\end{tabular}

To study the specific influence of the air content in hydraulic oil and the changes in ambient temperature on the performance of the FSS, it is necessary to combine the structural parameter analysis of the support cylinder and rolling diaphragm. The structural parameters of the designed support cylinder are listed in Table 2.

Table 2. The structural parameters of support cylinder.

\begin{tabular}{|c|c|c|c|}
\hline Name & Value & Name & Value \\
\hline $\begin{array}{c}\text { The installation height of small } \\
\text { diaphragm }\end{array}$ & $L_{1}=19.8 \mathrm{~mm}$ & $\begin{array}{l}\text { The width upper and lower } \\
\text { chambers }\end{array}$ & $W_{1}=2.6 \mathrm{~mm}$ \\
\hline $\begin{array}{l}\text { The height of upper and lower } \\
\text { chambers at } 0 \text { displacement }\end{array}$ & $L_{2}=2.4 \mathrm{~mm}$ & $\begin{array}{l}\text { The clearance between small } \\
\text { piston and cylinder wall }\end{array}$ & $W_{2}=11.0 \mathrm{~mm}$ \\
\hline $\begin{array}{c}\text { The installation height of large } \\
\text { diaphragm }\end{array}$ & $L_{3}=2.4 \mathrm{~mm}$ & $\begin{array}{l}\text { The clearance between large } \\
\text { piston and cylinder wall }\end{array}$ & $W_{3}=2.6 \mathrm{~mm}$ \\
\hline The thickness of large piston piece & $L_{4}=11.8 \mathrm{~mm}$ & Small piston radius & $W_{4}=6.6 \mathrm{~mm}$ \\
\hline The chamfer size of inlet and outlet & $L_{5}=1.1 \mathrm{~mm}$ & The length inlet and outlet & $W_{5}=2.0 \mathrm{~mm}$ \\
\hline The diameter of inlet and outlet & $L_{6}=15.75 \mathrm{~mm}$ & Total inlet length & $W_{6}=8.0 \mathrm{~mm}$ \\
\hline $\begin{array}{c}\text { The compression height of small } \\
\text { diaphragm }\end{array}$ & $L_{7}=14.0 \mathrm{~mm}$ & $\begin{array}{c}\text { The compression width small } \\
\text { diaphragm }\end{array}$ & $W_{7}=3.0 \mathrm{~mm}$ \\
\hline
\end{tabular}

The structural parameters of rolling diaphragm are shown in Table 3. 
Table 3. The structural parameters of rolling diaphragm.

\begin{tabular}{cccc}
\hline Name & Value & Name & Value \\
\hline The radius of piston chamfer & $R_{p}=1.6 \mathrm{~mm}$ & Shore hardness & $H_{s w}=40 \mathrm{HS}$ \\
Preformed height & $K=3.8 \mathrm{~mm}$ & The thickness of side wall & $W_{s w}=0.43 \mathrm{~mm}$ \\
Mounting diameter & $D_{p 1}=42 \mathrm{~mm}$ & The thickness of small & $W_{h 1}=0.635 \mathrm{~mm}$ \\
The modulus of elasticity & $D_{p 2}=78 \mathrm{~mm}$ & diaphragm & $W_{h 2}=0.675 \mathrm{~mm}$ \\
\hline
\end{tabular}

\subsection{Effect of Ambient Temperature Change on Volume under Different Air Contents}

When the support cylinder is filled with hydraulic oil, the actual volume of the upper and lower chambers will change with the change in air content, ambient temperature, and support pressure. To understand the specific correspondence between the influencing factors and affected factors, the volume of the upper and lower chambers of the support cylinder that can accommodate hydraulic oil are calculated separately. In the simulation, the percentage of air content was expressed by the volume fraction, which was varied from 0 to $10 \%$ in steps of $0.5 \%$, the temperature varied from $10{ }^{\circ} \mathrm{C}$ to $30{ }^{\circ} \mathrm{C}$ in steps of $0.01{ }^{\circ} \mathrm{C}$, and the support pressure varied from $0.05 \mathrm{MPa}$ to $1.00 \mathrm{MPa}$ in steps of $0.05 \mathrm{MPa}$. The mathematical model established by Equation (16) was simulated and analyzed in MATLAB, and the obtained simulation results are shown in Figure 4.

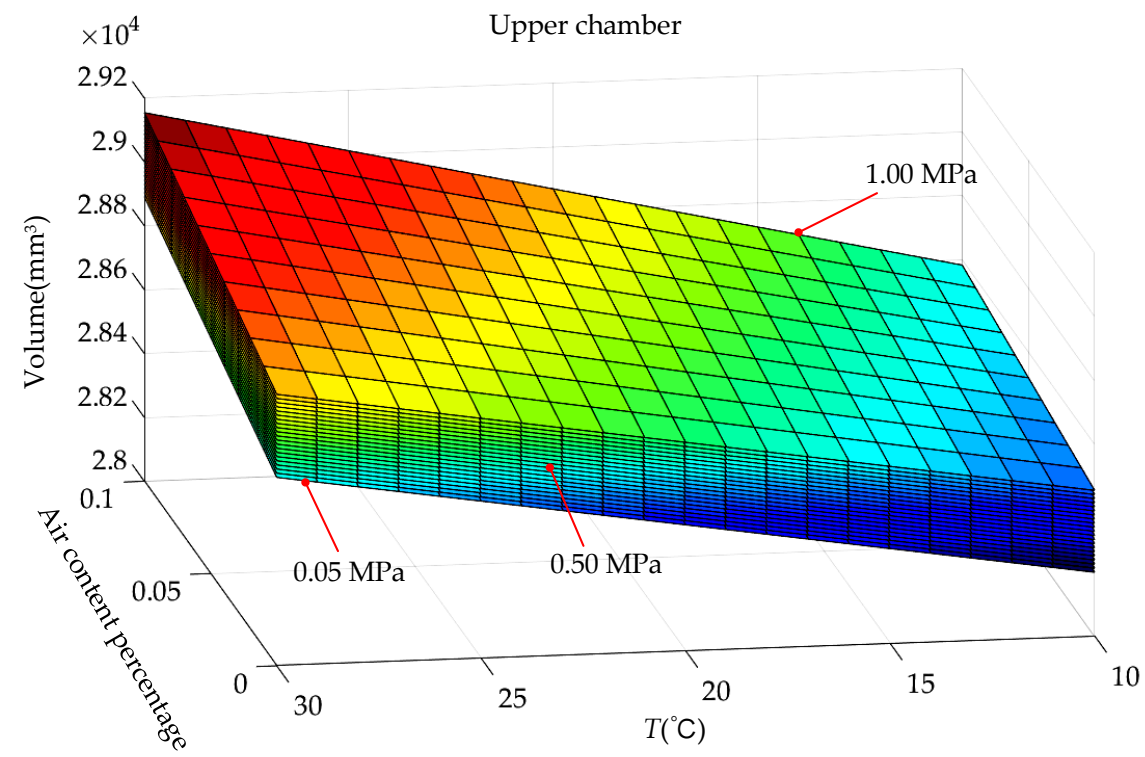

(a)

Figure 4. Cont. 


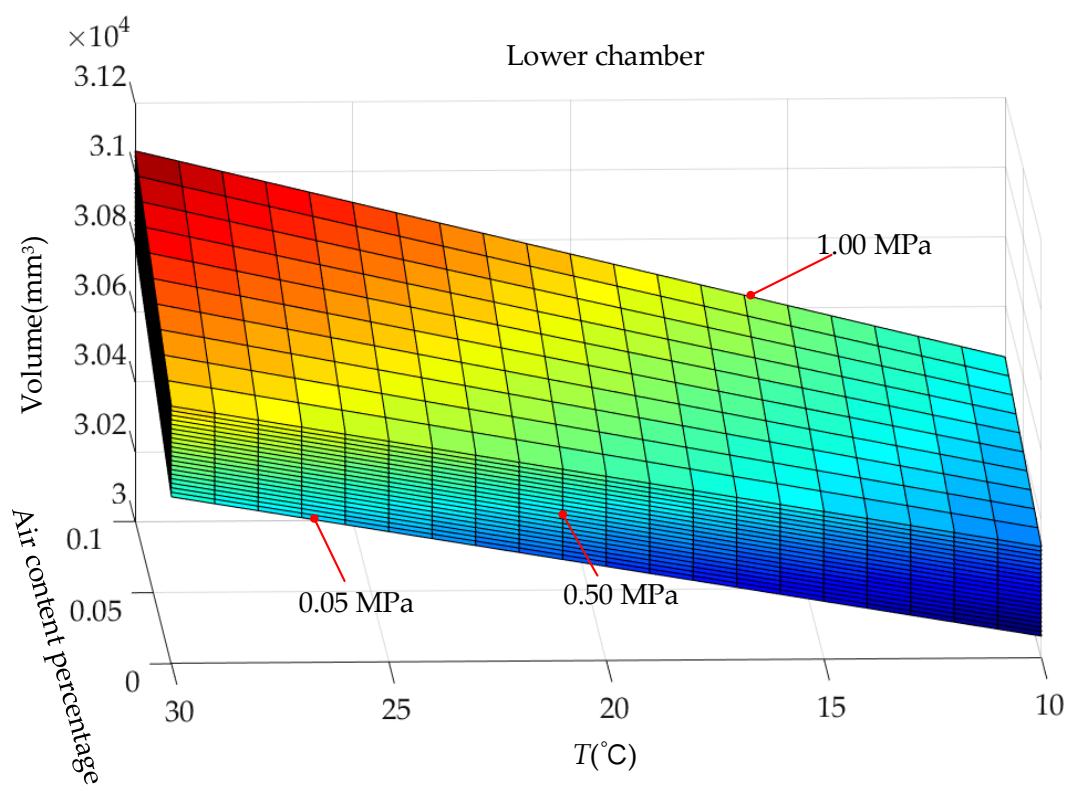

(b)

Figure 4. Variation of volume of upper and lower chambers with temperature and air content. (a) Volume of upper chamber. (b) Volume of lower chamber.

The relationship between the volume of the upper and lower chambers of the support cylinder and the ambient temperature, air content, and support pressure is shown in Figure $4 \mathrm{a}, \mathrm{b}$. It can be seen from the figure that the volume of the lower chamber of the support cylinder is still smaller than the volume of the upper chamber, and considering the weight of the internal and external piston parts, will cause the support shaft to move because of the asymmetric structure of the large rolling diaphragm. When the volume fraction of the air in the hydraulic oil and the ambient temperature are higher, the volume of the upper and lower chambers of the support cylinder will be larger. When the support pressure of the support system increases, the volume of the upper and lower chambers will increase, which is caused by the volume reduction of the rolling diaphragm when the pressure increases.

\subsection{Effect of Ambient Temperature Change on Support Height under Different Air Contents}

When the support shaft of the cylinder is from $-1.0 \mathrm{~mm}$ to $1.0 \mathrm{~mm}$, the volume of the upper and lower chambers of the support cylinder can be calculated by Equations (3) and (4). By simulating the effect of temperature change on volume under different air contents, the volume change in the upper and lower chambers can be calculated when the ambient temperature changes. Finally, in combination with Equation (2), when the ambient temperature and air content in the hydraulic oil are different, the change in the height of the support shaft under different working pressures can be calculated. The error is expressed by the difference between the set value and the actual measured value, and the errors mentioned below are calculated in this way.

The simulation results are shown in Figure 5. The smaller the working pressure of the FSS, the more sensitive the support height is to the change in air volume fraction and ambient temperature. There is no obvious stratification of the support height error caused by the pressure change, which indicates that the working pressure change has relatively little influence on the ambient temperature and air content of the support cylinder. When the ambient temperature and air volume fraction are both low or high, the support height error of the support cylinder is the most affected. When the air volume fraction is 0.1, the ambient temperature is $30^{\circ} \mathrm{C}$, and the support height can be reduced to $-0.073 \mathrm{~mm}$ in the negative direction owning to the influence of the ambient temperature and air 
content. At the same time, when the air volume fraction is 0 and the ambient temperature is $10{ }^{\circ} \mathrm{C}$, the support height can be increased to $0.083 \mathrm{~mm}$, owing to the influence of ambient temperature and air content. Therefore, to ensure the accuracy of the FSS, it is necessary to ensure that the range of fluctuation of the ambient temperature and air content in the working medium during the working process is as small as possible.

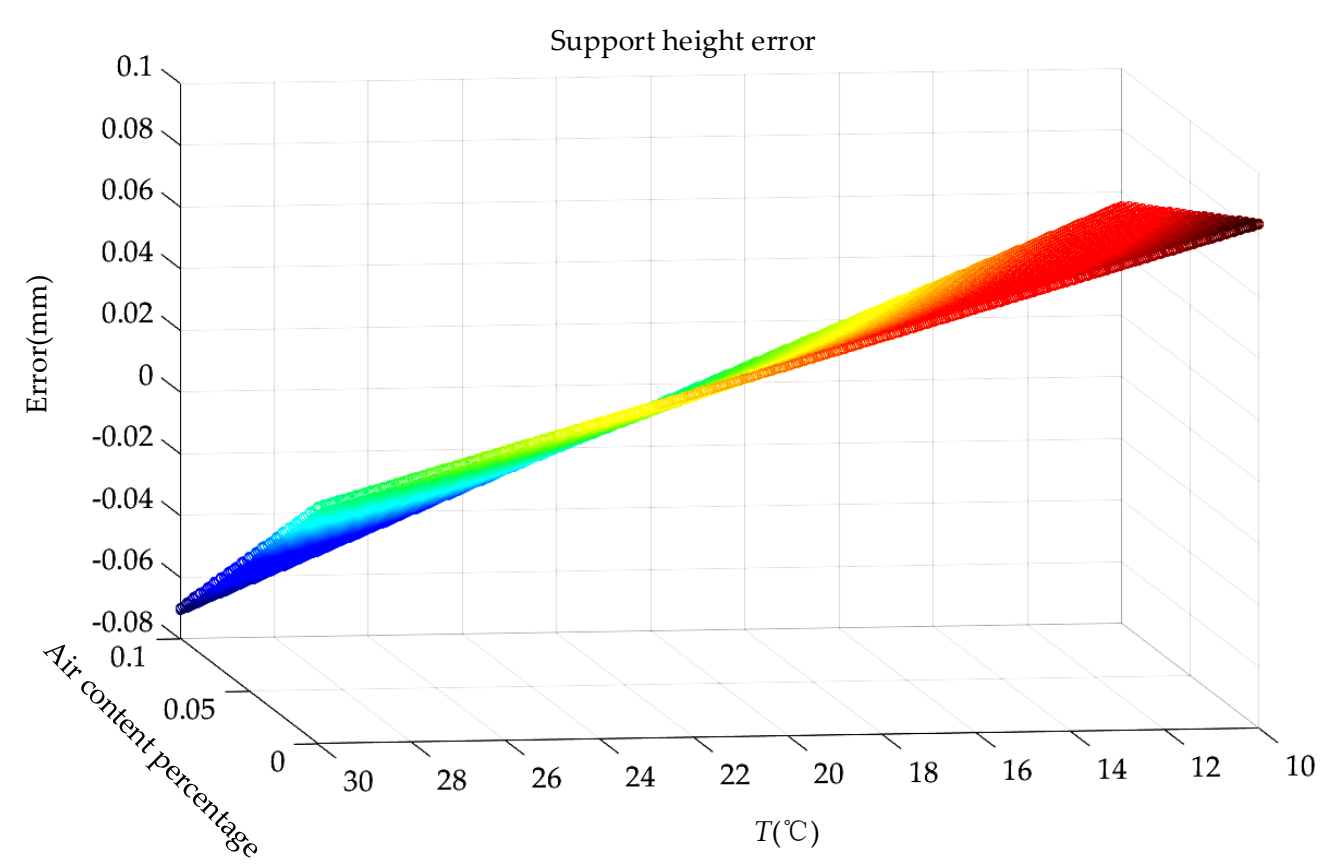

Figure 5. Effect of ambient temperature change on support height at different air contents.

\subsection{Effect of Ambient Temperature Change on Support Pressure under Different Air Contents}

The support pressures of the upper and lower chambers of the support cylinder under different working pressures are affected by changes in the ambient temperature and air content, as shown in Figure 6. From the simulation results, when the working pressure of the support cylinder is higher, the variation in ambient temperature and air content has a greater influence on the working pressure error of the upper and lower chambers. When the absolute working pressure of the support cylinder is 1.0 MPa, the maximum change in the upper chamber is $0.0075 \mathrm{MPa}$, and the maximum change in the lower chamber is $0.0074 \mathrm{MPa}$. When the absolute working pressure of the support cylinder is $0 \mathrm{MPa}$, the change in the upper chamber is $0.00125 \mathrm{MPa}$, and the affected change in the lower chamber is $0.00112 \mathrm{MPa}$. Therefore, from the standpoint of the influence of the working pressure of the support cylinder alone, the degree of influence on the support rigidity is low when the working pressure of the support cylinder is low. 


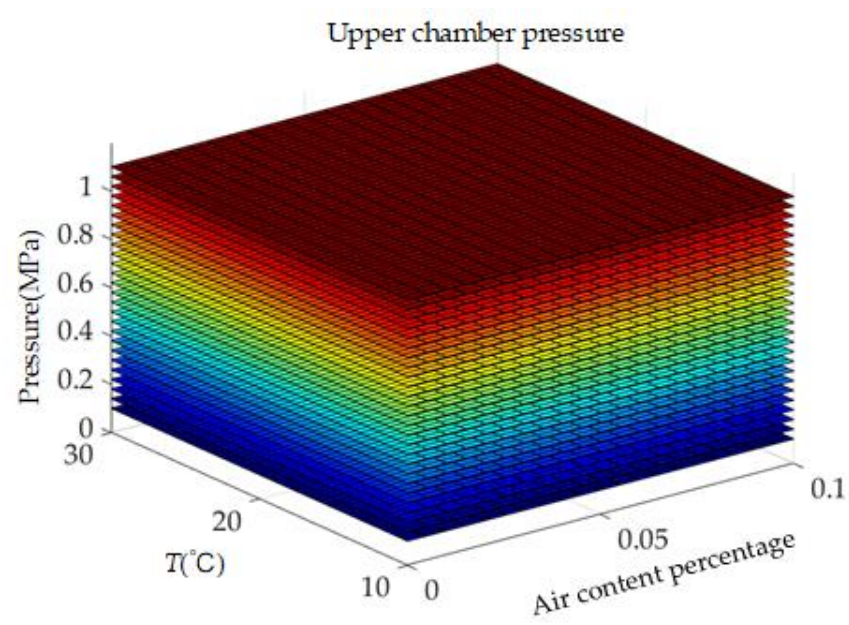

(a)

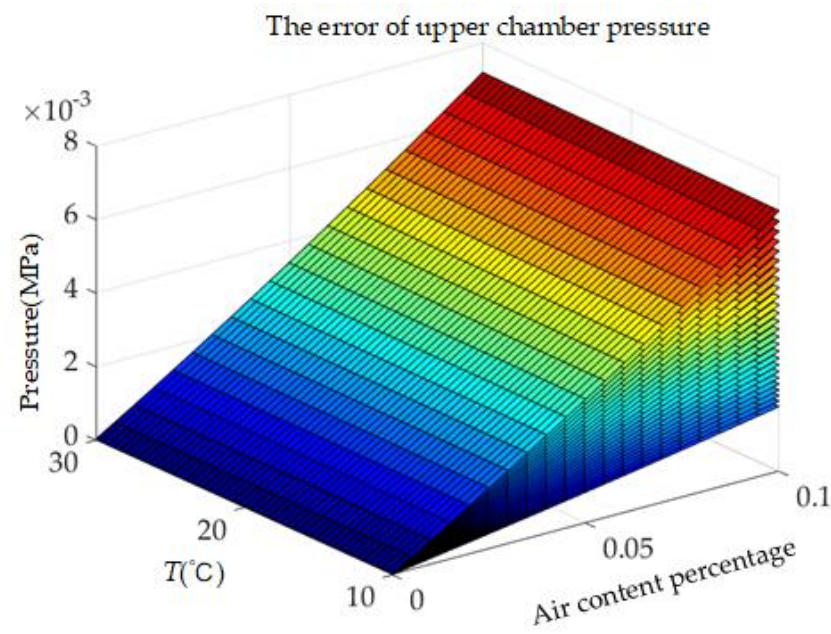

(c)

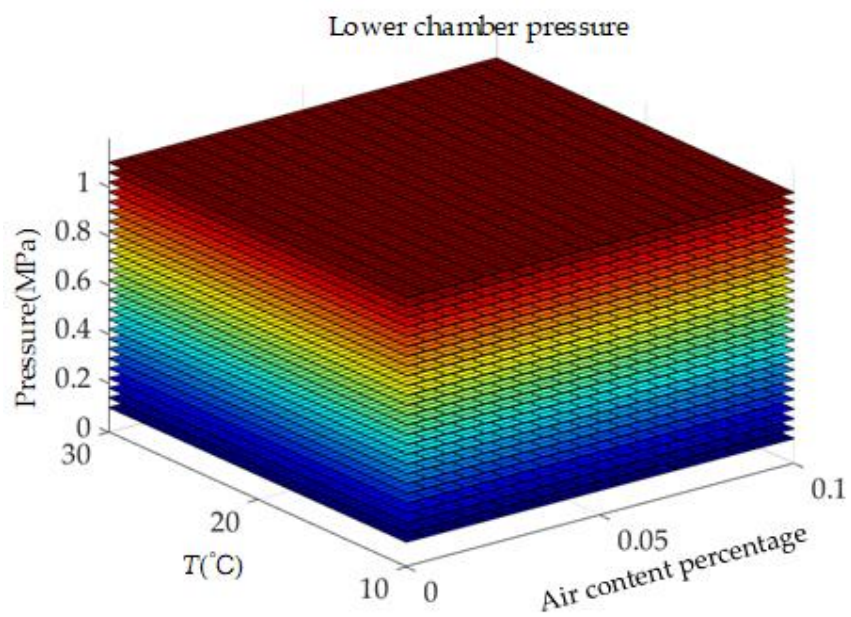

(b)

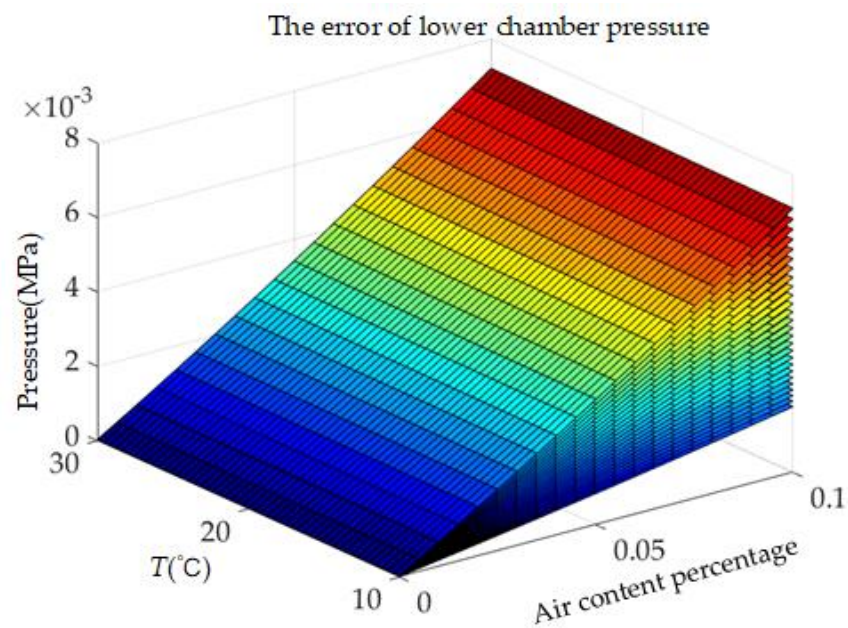

(d)

Figure 6. Support pressure is affected by change in ambient temperature and air content. (a) The pressure of upper chamber. (b) The pressure of lower chamber. (c) The error of upper chamber pressure. (d) The error of lower chamber pressure.

\section{Experiment Analysis}

The FSS is shown in Figure 7, and the rated support load of each support point is $100 \mathrm{~N}$. The weight of the force feedback sensors and connectors installed on each support point will share a part of the load. Therefore, the maximum weight of the FSS that theoretically supports the mirror is $300 \mathrm{~kg}$, which is generally sufficient for the support of an optical mirror with a diameter of $1250 \mathrm{~mm}$. The process of installing the optical mirror in the FSS is as follows: (i) Place the optical mirror on the 36 support points of the FSS and adjust the position to ensure that the center of rotation coincides with the support center of the FSS; (ii) calculate the support stiffness and support pressure that the FSS needs to maintain; (iii) pressurize the three sectors of the FSS to the rated pressure and theoretical height under the current working conditions and maintain them. It can be seen from the above that once the optical mirror and its process are determined, the support parameters of the FSS are determined. In addition, the amount of material removal during the processing of the optical mirror is extremely small compared to the weight of the mirror itself, the change in quality of the optical mirror can basically be ignored, and the rated load of the FSS during the working process does not change. Therefore, the change in the support height, support stiffness, and support pressure of the FSS caused by load changes is not considered during the experiment. 


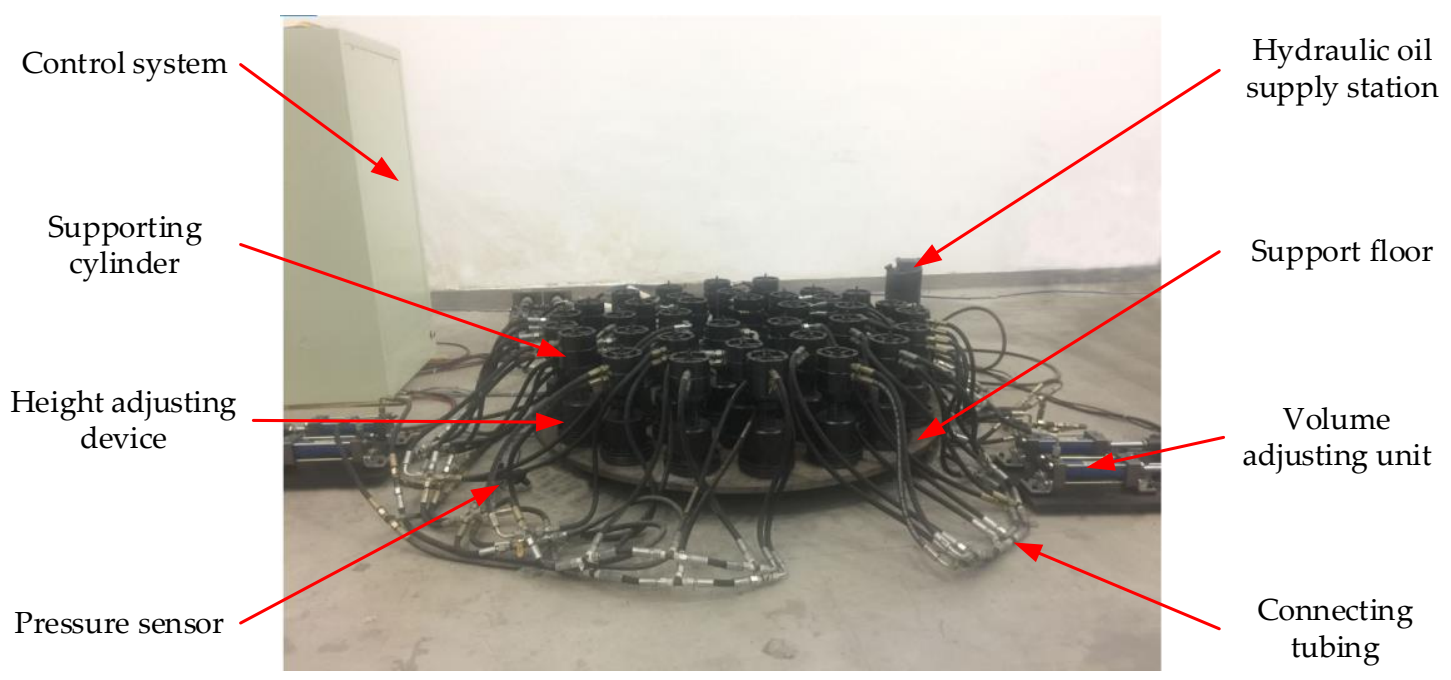

Figure 7. Flexible support system.

To determine the effect of ambient temperature and air content in hydraulic oil on the FSS in actual work, experimental studies on the influence of different ambient temperatures and air contents on the pressure of upper and lower chambers of the FSS and the support height and stiffness of the support cylinder were carried out. The 36 support cylinders were installed on the support floor, and high-strength tubing was used to connect the upper and lower chambers. The rated working pressure of the connecting tubing selected was 36.0 MPa, and the FSS was 0-1.0 MPa. During the experiment, the measured pressure of the FSS was $0.65 \mathrm{MPa}$, which was far less than the rated working pressure of the connecting tubing, and the influence of the deformation of the connecting tubing was ignored. Then, the hydraulic oil with unfiltered air and filtered air was tested on the FSS and hydraulic cylinder, respectively. When the hydraulic oil with unfiltered air was tested, the laboratory constant temperature controller was turned off after the hydraulic oil was injected, and the laboratory temperature changed with the ambient temperature. Then, the system pressure was increased to $0.65 \mathrm{MPa}$, and the FSS pressure and support height under this working pressure were evaluated with the change trend in ambient temperature. When hydraulic oil with filtered air was tested, first the air in the hydraulic oil was filtered through an air filter element. Then, the filtered hydraulic oil was injected into the FSS while ensuring that the hydraulic oil did not re-contact air during the work. Finally, the laboratory constant temperature controller was turned off so that the laboratory temperature changed with the ambient temperature. The main measuring equipment used in experiment is shown in Table 4 and the experimental site is shown in Figure 7.

Table 4. The main measuring equipment used in the experiment.

\begin{tabular}{cccc}
\hline Number & Name & Model & Accuracy \\
\hline 1 & Laser micrometer & LG-2 mm & $7.6 \mathrm{~nm}$ \\
2 & Pressure sensors & MIK-P3000 & $0.075 \%$ \\
3 & Force sensor & LKC-20 kg & $0.1 \%$ F.S. \\
4 & Temperature sensor & ML X 9016 & $0.02{ }^{\circ} \mathrm{C}$ \\
\hline
\end{tabular}

\subsection{Influence of Ambient Temperature and Air Content on the Support Height of Support Cylinder}

The FSS is composed of 36 support cylinders. It is not easy to measure 36 support cylinders at the same time when the support height of the support cylinder is affected by ambient temperature change. Therefore, only one support cylinder was tested to determine the change in the support shaft height of the support cylinder. During the experiment, hydraulic oil with unfiltered air and filtered air were injected into the support cylinder. Then, the constant temperature controller in the laboratory was turned off so that 
the laboratory temperature would change with ambient temperature. At the same time, the change in the height of the support shaft under different ambient temperatures was measured by a laser micrometer.

At the beginning of the experiment, the support pressure of the FSS was raised to $0.65 \mathrm{MPa}$ and the measured laboratory ambient temperature was $25.1^{\circ} \mathrm{C}$. The measured experimental data are shown in Figure 8. When the unfiltered hydraulic oil was injected, the change in the support height of the support cylinder with the change in ambient temperature was the same as the theoretical trend obtained by the simulation of the established mathematical model. However, the support height error of the support cylinder was lower than the theoretical value and was mostly lower than zero, ranging from -0.018 to $0.0012 \mathrm{~mm}$. This is because it is difficult to determine the actual air volume fraction in hydraulic oil, and the theoretical value of the air volume fraction was $2 \%$ as a reference. Therefore, the accuracy of the established mathematical model can be determined within the allowable range of error.

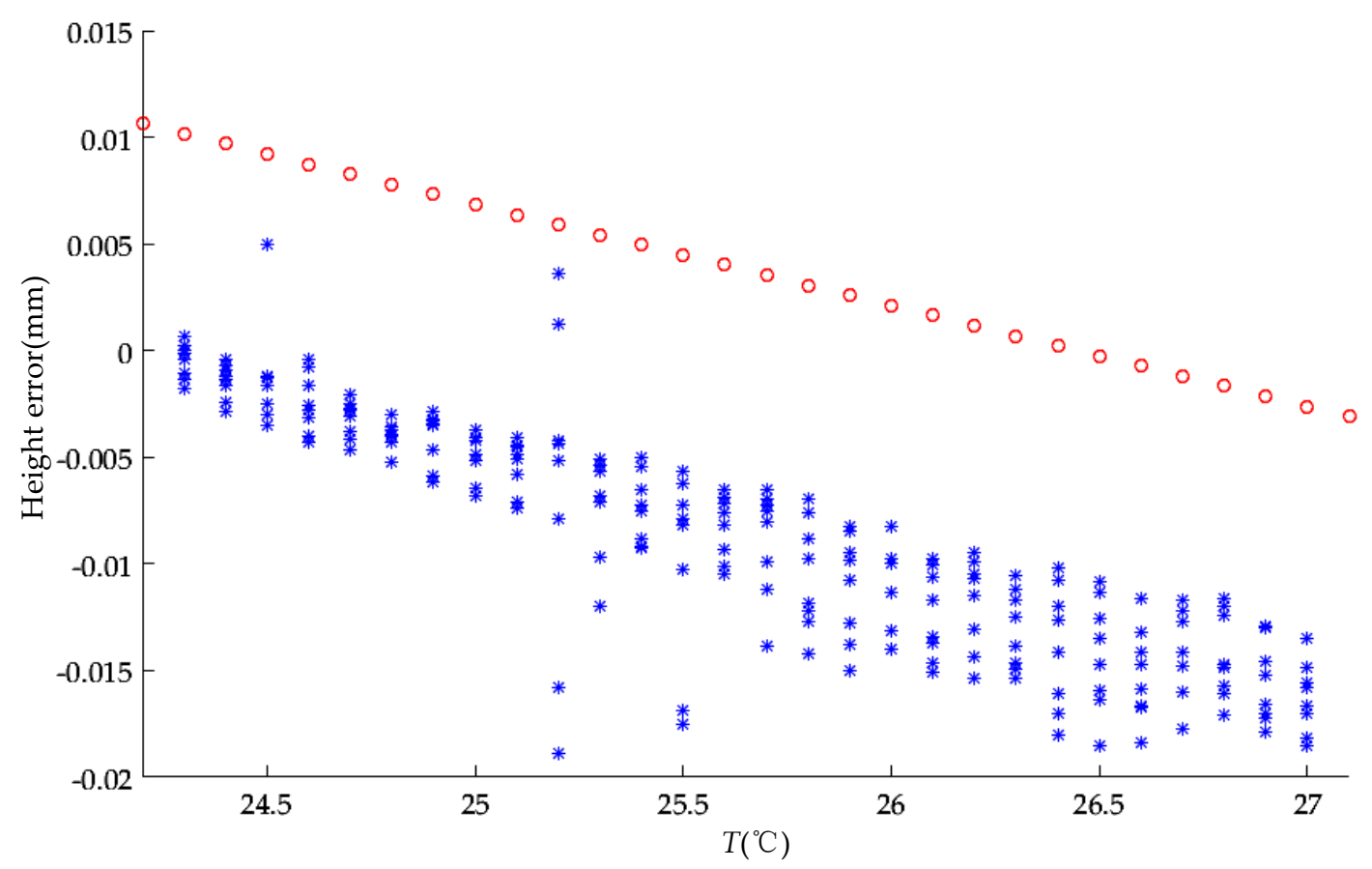

Figure 8. Support height error when injecting hydraulic oil with unfiltered air.

The filtered hydraulic oil was injected into the FSS and the error in the support height of the support cylinder with the change in ambient temperature is shown in Figure 9. It can be seen from the figure that when the laboratory temperature changed, the changes in the support height of the support cylinder were roughly the same as the theoretical value trend of the established mathematical model. The difference between the experimental and theoretical values is basically within $\pm 0.008 \mathrm{~mm}$, and the error is relatively large at individual moments. On the one hand, there may be a large error in the reading of the laser micrometer due to the presence of vibration during the measurement process. On the other hand, the measurement process lasts for a certain period, and the leakage phenomenon of the support cylinder under time space causes the support height error to change. Within the allowable range of error, the established mathematical model can accurately reflect the dynamic relationship between the performance parameters of the FSS and the ambient temperature and air content. It provides a theoretical basis for the subsequent research on the three-dimensional reconstruction of the spatial position of the optical mirror surface through the support points of the FSS, and also provides control parameters for height control during the processing. 


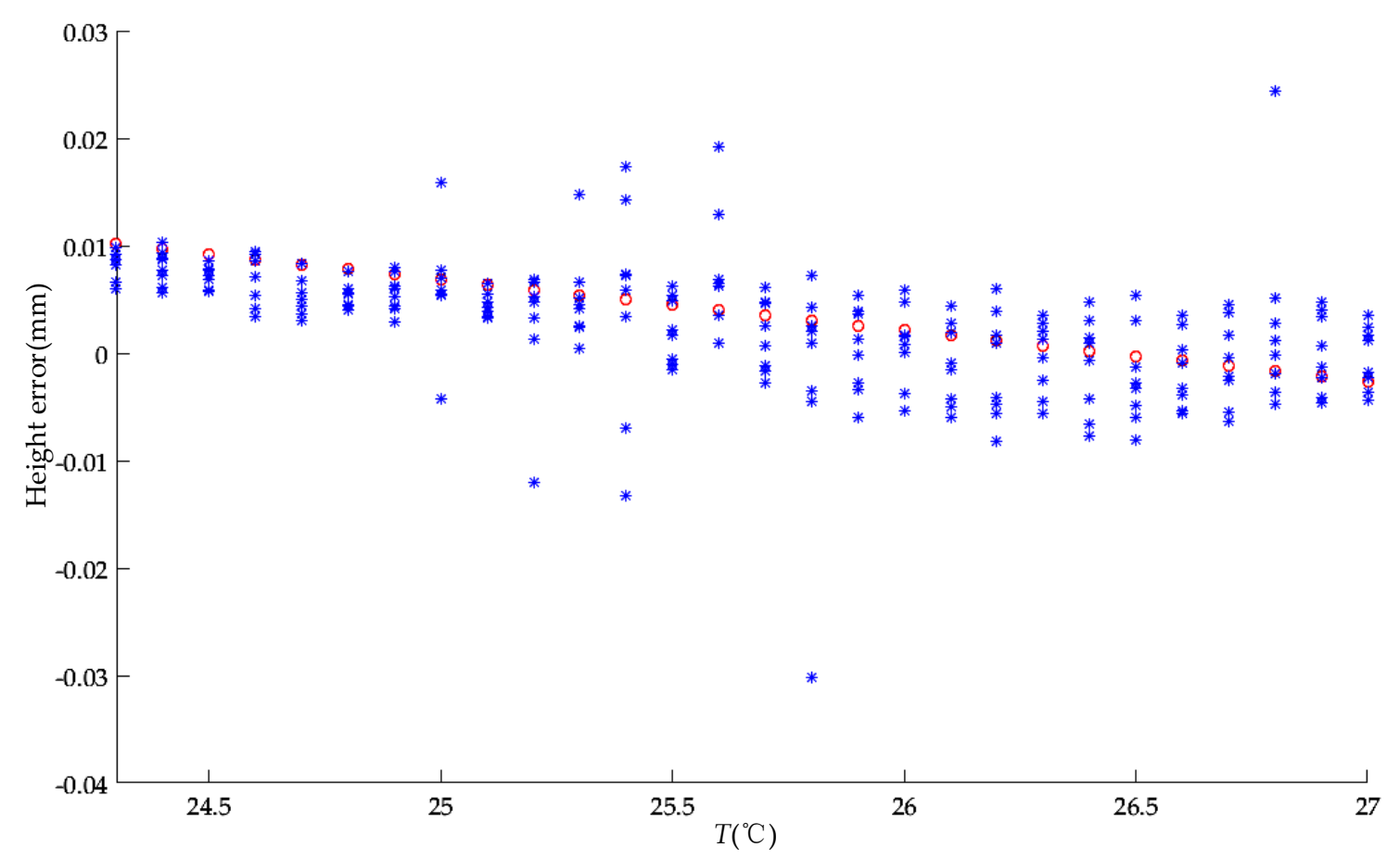

Figure 9. Support height error when injecting hydraulic oil with filtered air.

\subsection{Influence of Different Ambient Temperature and Air Content on the Pressure of Upper and Lower Chambers of FSS}

To determine the change pressure values of the upper and lower chambers of the FSS, pressure sensors were installed in the upper and lower chambers of the FSS to collect the pressure values in real time. To determine the compensation ability of the pressure transmitter under temperature changes, the electronic pressure transmitter and mechanical pressure gauge are connected to the pipeline to measure the system pressure.

It can be seen from Figure 10 that the pressure measured by the two sensors was roughly the same, and the resulting pressure deviation was a reading error. It can be concluded that the value measured by the pressure transmitter was accurate, and this data value was used as the measured data in the subsequent analysis process. During the experiment, the temperature in the laboratory fluctuated from $24^{\circ} \mathrm{C}$ to $27^{\circ} \mathrm{C}$, and the experiment lasted for $1640 \mathrm{~min}$. It can be seen from the collected pressure error of the upper and lower chambers of the FSS, after $900 \mathrm{~min}$ that, even if the temperature in the laboratory rises, the pressure error of the upper and lower chambers of the FSS is always negative and the deviation increases progressively. This phenomenon may be caused by leakage in the FSS, which leads to the loss of the working medium in the upper and lower chambers. Therefore, when analyzing the experimental data, the data collected before $900 \mathrm{~min}$ should be used for the analysis. The data collected in the first $900 \mathrm{~min}$ are shown in Figures 11 and 12, respectively. 


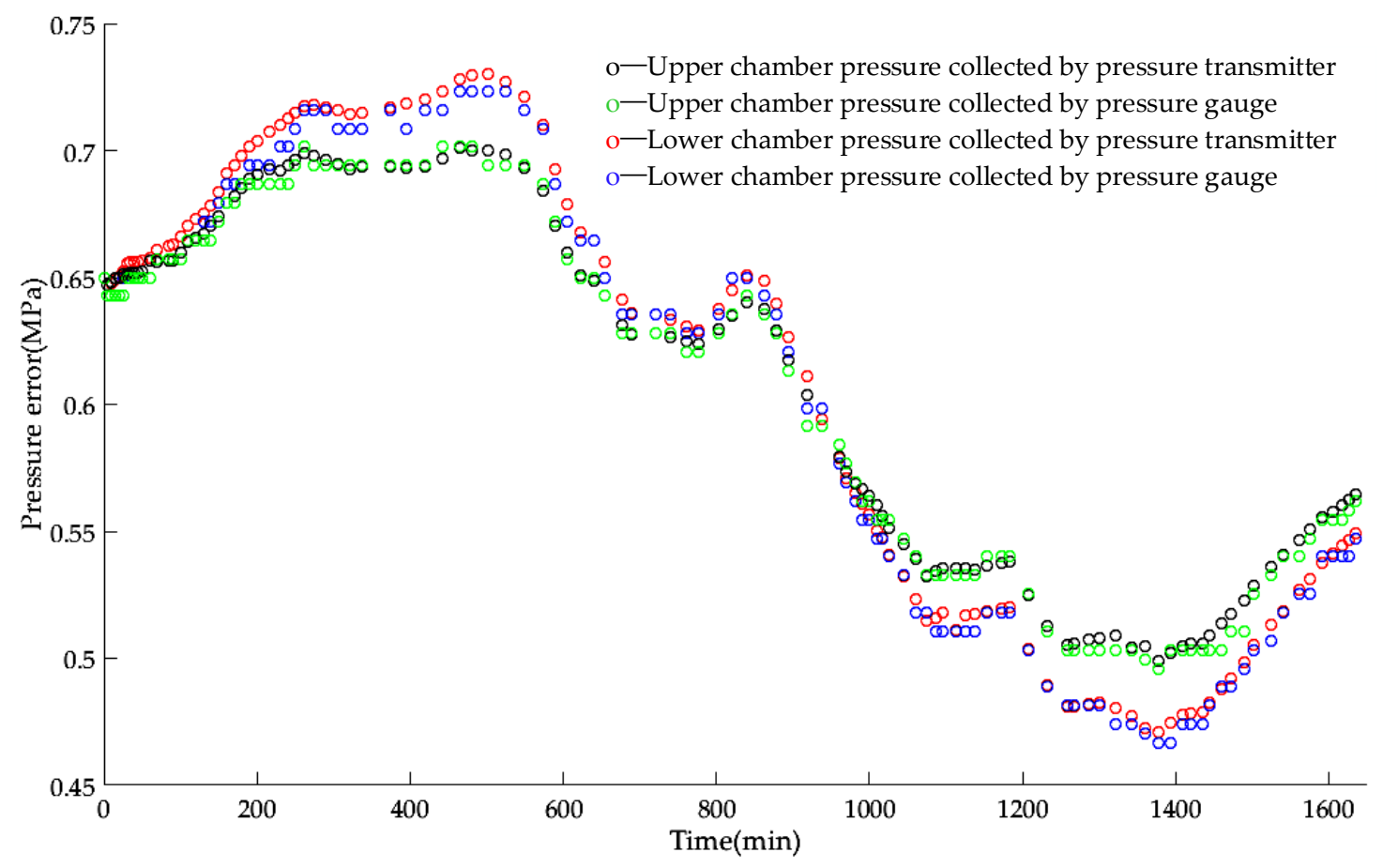

Figure 10. Data measured by electronic pressure transmitter and mechanical pressure gauge.

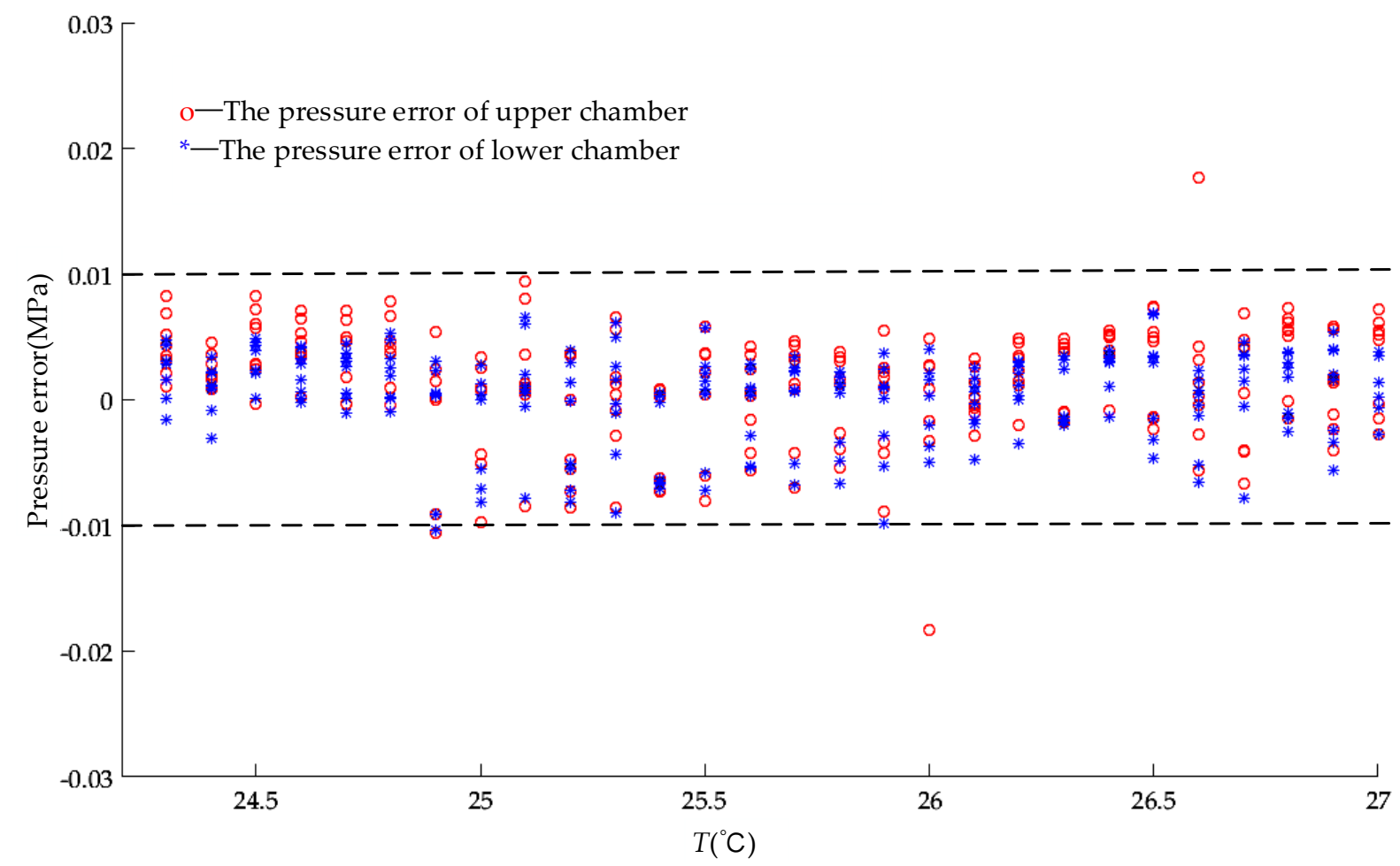

Figure 11. Pressure error of the upper and lower chambers when injecting unfiltered air. 


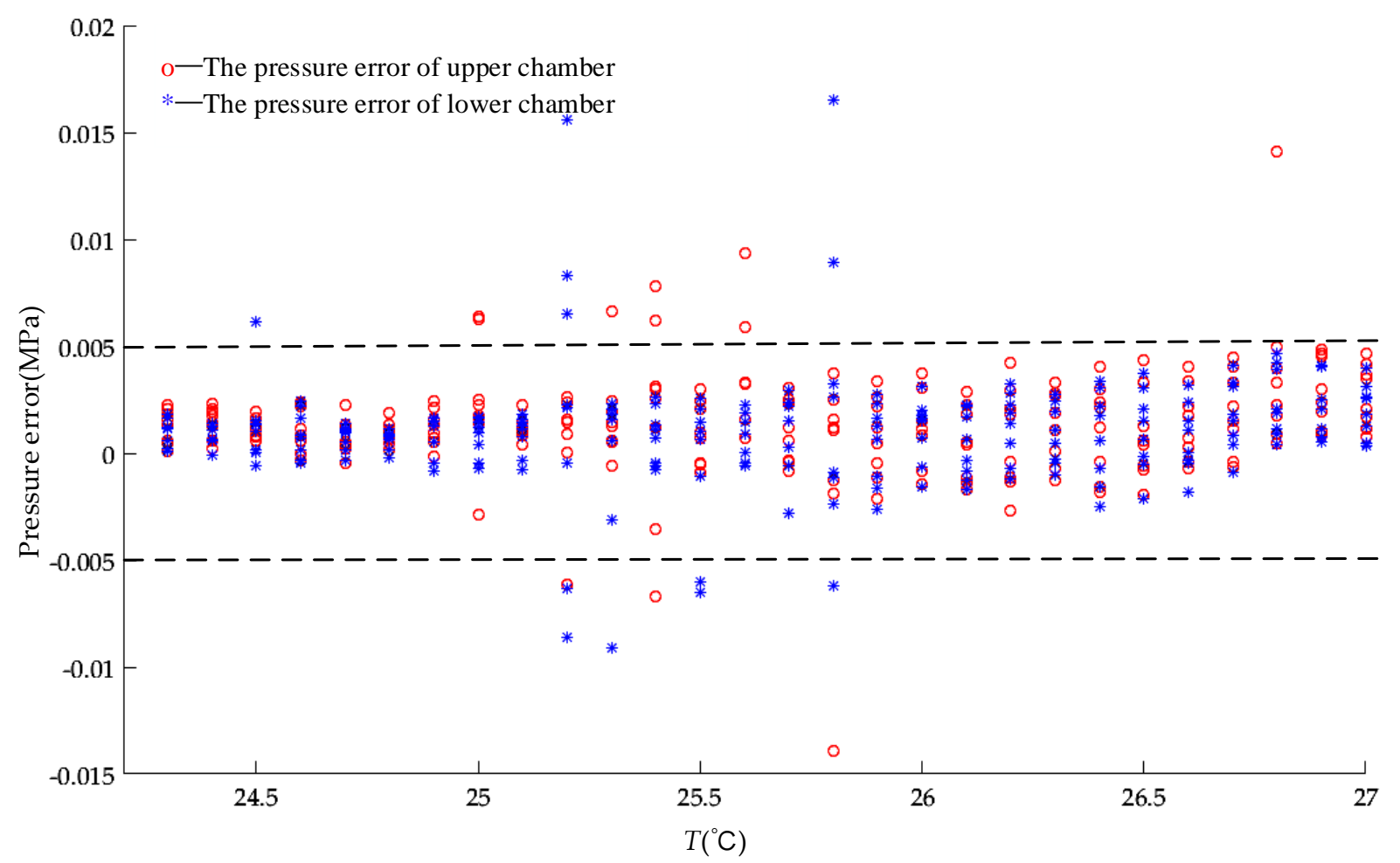

Figure 12. Pressure error of upper and lower chambers when injecting filtered air.

The pressure changes in the upper and lower chambers with changes in the ambient temperature when the hydraulic oil with unfiltered air and filtered air was injected into the FSS are shown in Figures 11 and 12. It can be seen from the data that the pressure error of the upper chamber is generally higher than that of the lower chamber. This is because, under the influence of gravity of the internal piston, external piston, support shaft, and lock nut of the support cylinder, the support piston will produce a downward displacement, resulting in the volume of the upper chamber being larger than the volume of the lower chamber. As a result, the pressure error in the upper chamber is always greater than the error generated by the lower chamber during ambient temperature change. It can be seen from the scatter plot that when the ambient temperature changed from $24.2{ }^{\circ} \mathrm{C}$ to $27^{\circ} \mathrm{C}$ and the working medium with unfiltered air was injected, the pressure error of the upper and lower chambers varied from $-0.011 \mathrm{MPa}$ to $0.01 \mathrm{MPa}$. When injecting the working medium with filtered air, the pressure error of the upper and lower chambers varied between $-0.0045 \mathrm{MPa}$ and $0.005 \mathrm{MPa}$. After the air was filtered, the pressure error in the support cylinder reduced significantly with the ambient temperature change, and the experimental results are roughly the same as the results of the simulation in Section 3.3, which further verifies the validity of the established mathematical model.

\subsection{Influence of Different Ambient Temperatures and Air Contents on the Support Stiffness of Support Cylinder}

Through the support pressure and support height errors collected as described in Sections 4.1 and 4.2, combined with Equations (23) and (24), the error of the support stiffness of the support cylinder can be obtained. The unfiltered air support stiffness error is shown in Figure 13. The support stiffness error of most test points is from $-280 \mathrm{~N} / \mathrm{mm}$ to $400 \mathrm{~N} / \mathrm{mm}$, and individual test points outside the interval may be caused by objective factors. The stiffness error of the filtered air support is shown in Figure 14. The support stiffness error of most test points in this case was between $-100 \mathrm{~N} / \mathrm{mm}$ and $190 \mathrm{~N} / \mathrm{mm}$. Compared with the unfiltered air working medium, the support stiffness error is greatly reduced, but it still varies randomly within the interval. This is due to the following systematic errors or random errors during the experiment. (i) During the experiment, 
the error of the support cylinder parts (such as machining error, nonlinear deformation caused by wire cutting of the beryllium bronze reed, and fatigue damage of the rolling diaphragm) led to inconsistent parameters such as the internal resistance of the support cylinder and the volume of the upper and lower chambers; (ii) during the installation of the support cylinder, the length and bending angle of the hydraulic pipes in the upper and lower chambers were not kept the same, which also caused errors in the FSS; (iii) during the experimental data collection process, due to the uneven heat dissipation source in the laboratory, the collected laboratory temperature was inconsistent with the hydraulic oil temperature; (iv) the FSS had leakage in the experiment and the leakage rate was unstable. These factors will cause errors in support stiffness, but the size of the error can be controlled within the range of -100 to $190 \mathrm{~N} / \mathrm{mm}$, which verifies the accuracy of the established mathematical model within the allowable range of error.

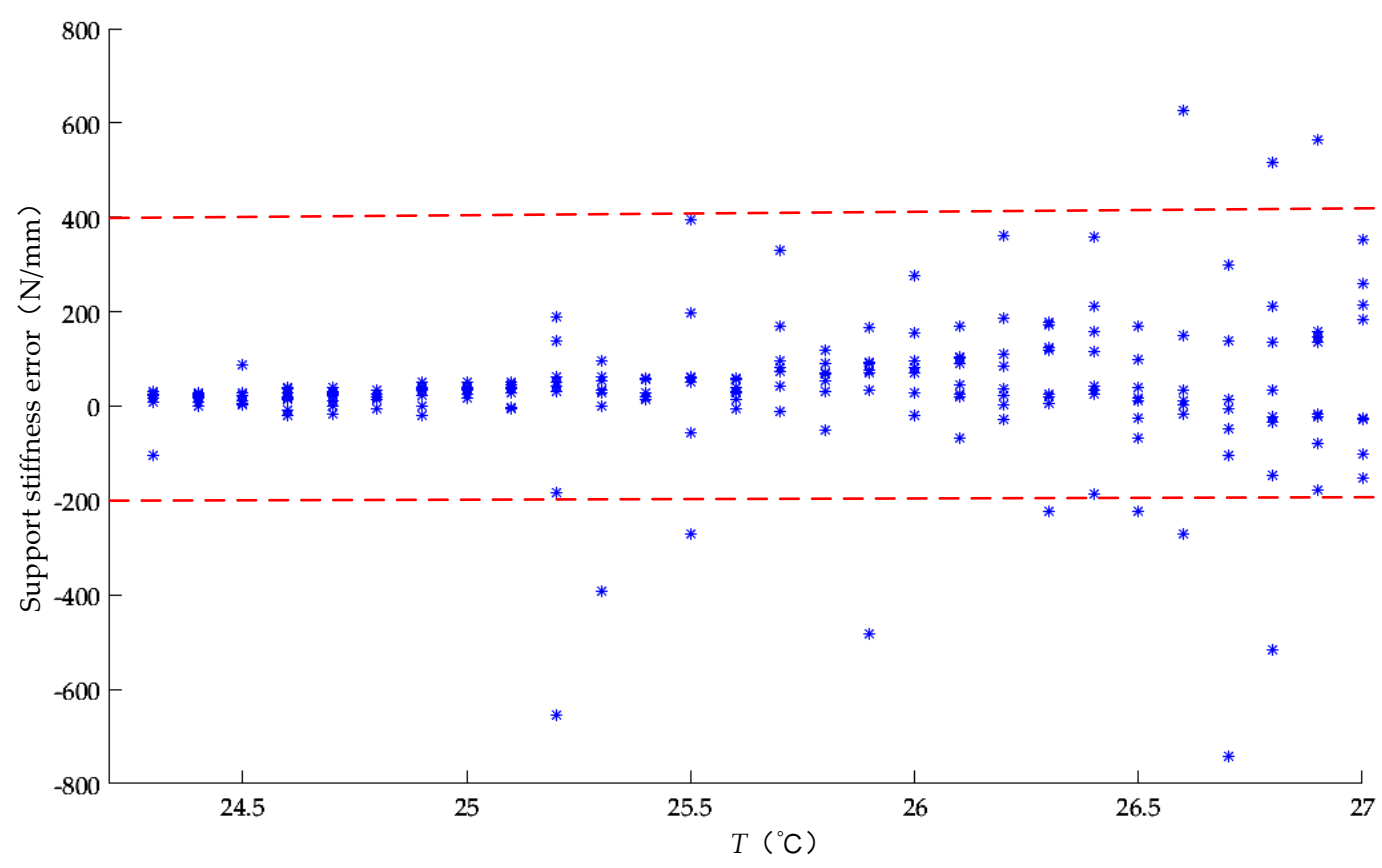

Figure 13. Support stiffness error with hydraulic oil with unfiltered air.

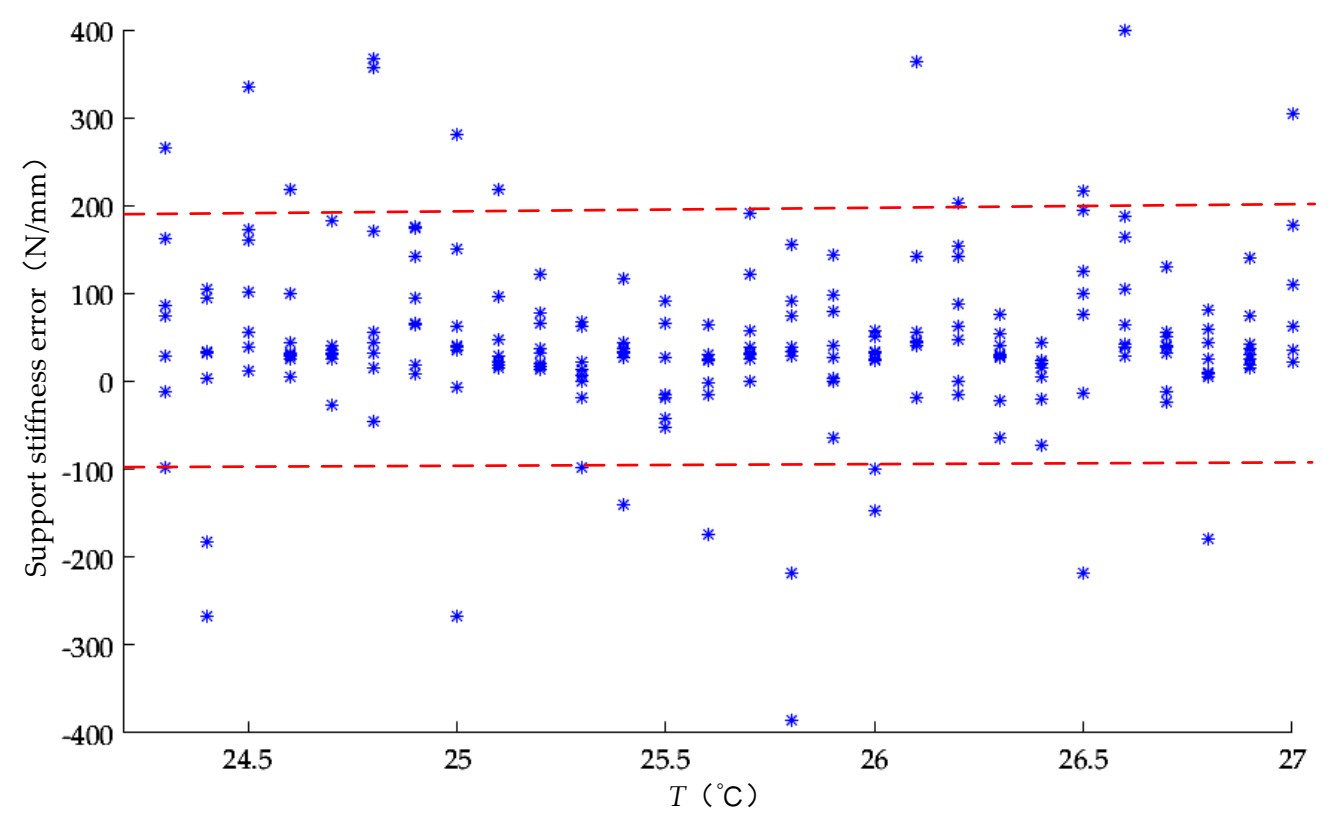

Figure 14. Support stiffness error with hydraulic oil with filtered air. 
Through the above experimental analysis, it can be seen that the support pressure error of the large-scale optical mirror FSS during the experiment was from $-0.0045 \mathrm{MPa}$ to $0.005 \mathrm{MPa}$, the support height error was from $-0.0045 \mathrm{MPa}$ to $0.005 \mathrm{MPa}$, and the support stiffness error ranged from $-100 \mathrm{~N} / \mathrm{mm}$ to $190 \mathrm{~N} / \mathrm{mm}$. These errors can be controlled within a certain range to meet the requirements of large-scale optical mirror processing. Applying this technology to the processing for large-scale optical mirrors can solve the shortcomings of the existing support system of large and non-adjustable support stiffness in the processing of optical mirrors. It can effectively unload the impact of robot motion accuracy and tool vibration during processing while protecting the optical mirror surface and more accurately controlling the amount of mirror removal.

\section{Conclusions}

The influence of support height, support pressure, and support stiffness of an FSS with variation in the ambient temperature and air content in hydraulic oil was studied. The mathematical models of the FSS and single support cylinder affected by the air content in the hydraulic oil and the ambient temperature were established, and the mathematical model was theoretically analyzed using MATLAB. From the simulation, it can be seen that: (i) The volume of the working medium increases as the temperature increases, and when the volume fraction of air in the working medium increases, the trend of its volume changes with temperature also shows an increase. (ii) Under different support pressures, the support height error of the FSS shows an increasing trend with the increase in ambient temperature and shows a decreasing trend with the increase in the air volume fraction in the working medium, and the change in support pressure has relatively little influence on support height. (iii) Under different support pressures, as the volume fraction of air content in the working medium and the ambient temperature increases, the pressure change in the upper and lower chambers of the FSS shows an increasing trend. When the support pressure of the FSS increases, the support pressure error of the upper and lower cylinders of the FSS is more sensitive to the influence of ambient temperature and air volume fraction. According to the above analysis results, the FSS and support cylinder were tested in two different cases where the air in the hydraulic oil was filtered and unfiltered. Through experimental analysis, it is concluded that the change trend of the support stiffness, support pressure, and support stiffness of the support cylinder is roughly the same as the theoretical values of the established mathematical model, and the validity of the established mathematical model is further verified within the allowable error range.

This study adopts the method of mathematical model-simulation-experiment to study the non-linear influence relationship between the ambient temperature and the air content in the working medium. The parameters of the model are modified by simulation analysis and combined with experimental parameters to verify that the established mathematical model can be consistent with the actual working conditions. The established mathematical model provides a theoretical basis for the subsequent modal analysis of the FSS and provides an experimental basis for the design of the controller and the study of error compensation control strategies. At the same time, this method can be extended to studies on the error mechanisms of other nonlinear, time-varying, uncertain, and externally disturbed systems.

Author Contributions: Methodology, software and writing, Z.J.; formal analysis, resources and supervision, G.C.; data curation, S.X.; project administration, W.G. All authors have read and agreed to the published version of the manuscript.

Funding: Financial support for this work, provided by the Priority Academic Program Development of Jiangsu Higher Education Institutions and the National Natural Science Foundation of China (Grant No. 91648105), are gratefully acknowledged.

Institutional Review Board Statement: This study does not involve humans or animals.

Informed Consent Statement: This study does not involve humans. 
Data Availability Statement: The simulation and experimental data used to support the findings of this study are available from the corresponding author upon request.

Conflicts of Interest: The authors declare that there are no conflicts of interest regarding the publication of this paper.

\section{References}

1. Lian, P.; Wang, C.; Xue, S.; Xu, Q.; Shi, Y.; Jia, Y.; Xiang, B.; Wang, Y.; Yan, Y. Surface adjustment strategy for a large radio telescope with adjustable dual reflectors. IET Microw. Antennas Propag. 2019, 13, 2669-2677. [CrossRef]

2. Ma, D.L. Recommended conceptual optical system design for China's large optical-infrared telescope (LOT). Opt. Express 2018, 26, 108-119. [CrossRef] [PubMed]

3. Fan, J.; Wu, Q.; Chen, B.; Liu, L.; Chen, L. Optical Design of the Goaly3 Multi-Mirror Telescope System with a Wide Field of View. Appl. Sci. 2021, 11, 1200. [CrossRef]

4. Tan, Y.; Wang, J.; Ren, G.; Xie, Z.; He, B. Thermal control analysis of a primary mirror for large-aperture telescope. J. Korean Phys. Soc. 2017, 71, 28-36. [CrossRef]

5. Kong, Y.; Cheng, G.; Guo, F.; Gu, W.; Zhang, L. Inertia matching analysis of a 5-DOF hybrid optical machining manipulator. J. Mech. Sci. Technol. 2019, 33, 4991-5002. [CrossRef]

6. Je, S.; Yeo, I.; Jung, S.K.; Kim, J.D.; Lee, K.S.; Kim, G.H. Development of spiral grinding process technology for glass lens hole machining. Int. J. Precis. Eng. Manuf. 2019, 20, 1877-1883. [CrossRef]

7. Jin, Z.; Cheng, G.; Chen, S.; Guo, F. Human-machine-environment information fusion and control compensation strategy for large optical mirror processing system. Proc. Inst. Mech. Eng. Part C J. Mech. Eng. Sci. 2020. [CrossRef]

8. Niu, Y.; Chang, J.; Shen, B.; Chen, W. Optical design of a null test of a synthetic reflective mirror based on the subaperture test method. Opt. Eng. 2017, 56, 094106. [CrossRef]

9. Su, D.Q.; Liang, M.; Yuan, X.; Bai, H.; Cui, X. The optical system of the proposed Chinese 12-m optical/infrared telescope. Mon. Not. Roy. Astron. Soc. 2017, 469, 3792-3801. [CrossRef]

10. Yan, J.; Peng, Y.; Nie, D. A Method for the Installation Measurement and Alignment of a Mirror Unit in the Solar Dish Concentrator. Appl. Sci. 2020, 10, 1511. [CrossRef]

11. Hu, H.; Luo, X.; Liu, Z.; Zhang, X.; Xue, D.; Zhao, H. Designing a hydraulic support system for large monolithic mirror's precise in-situ testing-polishing iteration. Opt. Express 2019, 27, 3746-3760. [CrossRef] [PubMed]

12. Huo, T.L.; Yu, J.J.; Zhao, H.Z. Design of a kinematic flexure mount for precision instruments based on stiffness characteristics of flexural pivot. Mech. Mach. Theory 2020, 50, 103868. [CrossRef]

13. Yu, F.A.; Xu, S.Y. Flexible support structure based on spring principle for a high precision reflecting mirror. Optik 2020, 207, 164341. [CrossRef]

14. Zhang, L.; Wang, W.; Wang, J.; Guo, P.; Hao, L.; Zhang, L.; Liang, H. Design and analysis for the multi-point flexible support structure of large and precision lens. Optik 2019, 193, 162966. [CrossRef]

15. Jafari, M.; Mobayen, S. Second-order sliding set design for a class of uncertain nonlinear systems with disturbances: An LMI approach. Math. Comput. Simul. 2019, 156, 110-125. [CrossRef]

16. Mofid, O.; Mobayen, S.; Khooban, M.H. Sliding mode disturbance observer control based on adaptive synchronization in a class of fractional-order chaotic systems. Int. J. Adapt. Control Signal Process. 2019, 33, 462-474. [CrossRef]

17. Gholizadeh, H.; Bitner, D.; Burton, R.; Schoenau, G. Modeling and experimental validation of the effective bulk modulus of a mixture of hydraulic oil and air. J. Dyn. Syst. Meas. Control-Trans. ASME 2014, 136, 051013. [CrossRef]

18. Kim, S.; Murrenhoff, H. Measurement of effective bulk modulus for hydraulic oil at low pressure. J. Fluids Eng.-Trans. ASME 2012, 134, 021201. [CrossRef]

19. Burecek, A.; Hruzik, L.; Vasina, M. Determination of undissolved air content in oil by means of a compression method. Strojniski Vestn. J. Mech. Eng. 2015, 61, 477-485. [CrossRef]

20. Zhou, J.J.; Hu, J.B.; Yuan, S.H. Modeling bubble evolution in air-oil mixture with a simplified method. Proc. Inst. Mech. Eng. Part C J. Eng. Mech. Eng. Sci. 2016, 230, 2865-2871. [CrossRef]

21. Mobayen, S. Optimal LMI-based state feedback stabilizer for uncertain nonlinear systems with time-varying uncertainties and disturbances. Complexity 2016, 21, 356-362. [CrossRef]

22. Mobayen, S.; Tchier, F. Nonsingular fast terminal sliding-mode stabilizer for a class of uncertain nonlinear systems based on disturbance observer. Sci. Iran 2017, 24, 1410-1418. [CrossRef]

23. Yuan, X.M.; Zhu, X.; Wang, C. Research on theoretical model of dynamic bulk modulus of gas-containing hydraulic oil. IEEE Access 2019, 7, 178413-178422. [CrossRef]

24. Righettini, P.; Strada, R.; Valilou, S.; KhademOlama, E. Nonlinear model of a servo-hydraulic shaking table with dynamic model of effective bulk modulus. Mech. Syst. Signal Proc. 2018, 110, 248-259. [CrossRef] 